\title{
Efficient Modeling of Transmission Lines With Electromagnetic Wave Coupling by Using the Finite Difference Quadrature Method
}

\author{
Qinwei Xu, Member, IEEE, and Pinaki Mazumder, Fellow, IEEE
}

\begin{abstract}
This paper proposes an efficient numerical technique, called the finite difference quadrature (FDQ) method, to model the transmission line with radiated electromagnetic (EM) wave noise coupling. A discrete modeling approach, the FDQ method adapts coarse grid points along the transmission line to compute the finite difference between adjacent grid points. A global approximation scheme is formulated in the form of a weighted sum of quantities beyond the local grid points. Unlike the Gaussian quadrature method that computes numerical integrals by using global approximation framework, the FDQ method uses a global quadrature method to construct the approximation schemes for the computation of, however, numerical finite differences. As a global approximation technique, the FDQ method has superior numerical dispersion to the finite difference (FD) method, and, therefore, needs much sparser grid points than the FD method to achieve comparable accuracy. Equivalent voltage and current sources are derived, exciting the transmission line at the grid points. Equivalent circuit models are consequently derived to represent the transmission line subject to radiated electromagnetic wave noise. The FDQ-based equivalent models can be integrated into a simulator like SPICE.
\end{abstract}

Index Terms-Electromagnetic (EM) wave illuminating, external field coupling, finite difference quadrature (FDQ) method, interconnect modeling, transient simulation, transmission lines (TL).

\section{INTRODUCTION}

$\mathbf{E}$ LECTROMAGNETIC interference (EMI) problems have been a great concern in high-speed digital systems and plenty of works have been done to handle the electromagnetic compatibility (EMC). Depending on different propagation approaches, there are conducted EMI noise, capacitive/inductive coupling EMI noise, and radiated electromagnetic (EM) wave noise. Conducted and coupling EMI problems have been studied most in the literature since fast operation and large integration scale started making the interconnect effect an important issue in high-speed systems two decades ago. In addition to having the on-chip and on-board effects of delay, crosstalk, and reflection, electrically long interconnects pose the antenna effect,

Manuscript received July 10, 2004. This work was supported in part by a Multidisciplinary University Research Initiative (MURI) grant and also by an Office of Naval Research (ONR) grant under the Dual-Use Program.

Q. Xu is with Research and Development Team of Electronic Design Automation Tools, Cadence Design Systems, San Jose, CA 95134 USA (e-mail: qwxu@umich.edu).

P. Mazumder is with the Department of Electrical Engineering and Computer Science, the University of Michigan, Ann Arbor, MI 48109 USA (e-mail: mazum@eecs.umich.edu).

Digital Object Identifier 10.1109/TVLSI.2007.904105 when they receive considerable dose of incident electromagnetic (EM) waves emitted by other electronic devices [1]. Fast clocking rate and short rise time result in signals with wavelengths comparable to interconnect sizes that increase the radiation efficiency of the conducting traces, while the shrinking feature size and the increasing integration scale lead to higher EMI susceptibility among the circuit parts. The antenna-effect EMI problem becomes a more serious challenge to signal integrity with progressive $V_{\mathrm{DD}}$ down-scaling.

With the traditional EMC problems being involved most with cabling, there are a few scenarios in which the external EM waves are coupled to transmission lines (TL) in the high-speed systems. The interconnects on printed circuit board (PCB) illuminated by EM waves are in the first scenario. As the PCB routing generally involves long interconnects, the EM wave coupling most likely happens in this case, which is in the category of PCB level EMI problem. In the second scenario the packaging structures, like the pad/pin and the leadframe, are subject to EM wave coupling. The packaging structures have electrically large sizes to pick up the illuminating EM wave and the resultant EMI noise travels inside the chips and interferes with signals. This situation is in the packaging level EMI problems. In the third scenario, the on-chip long interconnects, like power/ground lines and clocking lines, have the antenna effect in which the induced voltages and currents due to EM wave pose the sources of noise. Also, in the large array structures, like RAM/ROM, the horizontal and vertical data tracks are long and, therefore, may be sensitive to the external interference. These cases are the on-chip level EMI problems.

EM wave coupling to transmission lines can happen at any level as discussed before. The problem has been handled by using 3-D full-wave solvers like finite-difference time-domain (FDTD) methods. In the circuit oriented EMI application, FDTD models the lumped devices as grid or subgrid elements having the explicit integration scheme as FDTD required [2]. Following the Courant constraints, the FDTD theoretically gives accurate results as it directly simulates the wave phenomena represented by electric field and magnetic field. However, in view of circuit design and circuit simulation, direct full-wave technique, usually having 3-D scale, is computationally expensive and, therefore, prohibitive in most of the cases. This is especially true when handling the on-chip problem where the operation frequencies are much higher than those on off-chip or PCB. Furthermore, full-wave solvers may suffer from numerical instability when incorporated into circuit simulators, due to its tiny step size determined by the Courant condition. 
On the other hand, the transmission line representation of the interconnects is shown to be still valid even at a relatively high frequency, provided that the return path is well designed, which is always observed in practical CMOS and PCB designs. This fact justifies the situation that equivalent circuit modeling, based on the quasi-TEM assumption, is one of the mainstream approaches to solve the problem of external field coupling to transmission lines [3], [4]. In the equivalent circuit modeling, the incident EM waves illuminating the transmission lines are equivalently modeled as either lumped sources or terminal sources. The effects of the incident field are represented as forcing functions added into the transmission line equations, and, consequently, they are formulated and incorporated into the circuit simulators altogether with other devices. Compared to the field solvers, the equivalent circuit approach is computationally efficient and numerically accurate as long as the quasi-TEM assumption is valid.

In the equivalent lumped source approach [5], [6] that is mathematically based on the finite difference (FD) methods, the transmission lines are segmented into small sections, each of which is represented by lumped elements, and the equivalent sources of the external EM wave are added at each small section. As the FD methods have low-order accuracy, the electrical length of each section has to be a considerably small fraction $(1 / 12-1 / 20)$ of the minimum wave length of the signal; therefore, the equivalent models consist of an excessive number of lumped elements. On the contrary, the equivalent terminal source approach [7], [8] adapts the equivalent sources of the external EM wave only at the input and the output ends of the model by integrating the EM wave effect along the entire transmission line, therefore, reduces the number of the internal nodes of the equivalent models. Terminal source modeling methods, e.g., the method of characteristics (MC) [7] and the Padé approximation of the exponential matrices [8], are efficient in some cases since they bypass the internal grid point process. However, as the terminal source modeling methods lack the flexibility of numerical computation due to their use of integral involving transcendental functions, their applications are limited either to lossless transmission lines or uniform transmission lines.

In spite of the numerical flexibility of FD methods, the problem with FD modeling is that, although we are only interested in a small number of grid points, especially the end points, the grid points have to be dense enough to accurately represent the derivatives along the transmission lines, which leads to large sparse matrices. The drawback of low-order finite methods can be removed by using the high-order finite methods or pseudospectral methods [9], [10]. The mathematical fundamental of finite difference schemes is the Taylor series expansion. The scheme of a low-order finite method is determined by low-order Taylor series, while the scheme of high-order finite method is determined by high-order Taylor series. In general, the high-order schemes have a high order of truncation error. Thus, to achieve the same order of accuracy, the mesh size used by the high-order schemes can be much smaller than that used by low-order schemes. As a result, the high-order schemes can obtain accurate numerical solutions using much fewer mesh points.
On the other hand, numerical integration (quadrature) is more stable and reliable than differentiation. An integral approximation framework includes the global grid points over the entire domain, while a differential one includes only the local grid points. Given an electromagnetic problem, integral approaches, like Gaussian quadrature, generally give more accurate solutions than the differentiation approaches that use local approximation [11]. In this paper, the finite difference quadrature (FDQ) method is proposed to model transmission lines. The idea of the FDQ method is to quickly compute the finite difference between two neighboring grid points by estimating a weighted linear sum of derivatives at a small set of global grid points belonging to the domain. The weighted linear sum is like the numerical integral in the Gaussian quadrature method, yet it is to compute the finite differences rather than the integrals.

This paper develops the FDQ method to model transmission lines and the radiated EM wave coupling in the following steps. At first, the effects of the incident EM wave are mathematically represented by forcing functions on the right-hand side of the Telegrapher's equations governing the transmission lines. Starting from transmission line equations in the frequency domain, FDQ discretizes the ordinary differential equations (ODEs) as algebraic equations which give the discrete model of the transmission lines. Due to the globality of its approximation scheme, FDQ has remarkably improved numerical dispersion, and high accuracy can be obtained using a small number of grid points, which reduces the modeling complexity. The radiated EM wave noises are then modeled as equivalent sources exciting at the grid points. The number of the equivalent sources, which is the same as that of the grid points, is much less than the number of grid points in the FD method, and, therefore, maintains a balance between accuracy and efficiency. The transmission line is thus modeled as a multiport device and its time domain model is obtained by applying inverse Laplace transform.

The organization of this paper is as follows. In Section II, the quasi- TEM formulations of transmission lines with the incident EM waves (represented by the forcing functions) are briefly reviewed. The mechanism of the FDQ method is developed and its numerical properties are shown in Section III. In Section IV, numerical examples are presented and the results by a variety of FDQ methods have been compared with the results obtained using HSPICE.

\section{QUASI-TEM FORMULATIONS OF EM WAVE COUPLING TO TL}

\section{A. Formulations Under Quasi-TEM Assumption}

The quasi-TEM assumption of TLs is equivalent to the condition that the dimensions of TLs cross-sectional sizes are much smaller than a wavelength $\lambda$ of the external EM wave. Under such a condition, the principal propagation mode of the TLs is TEM, and can be accurately described by the Telegrapher's equations. Theoretical analysis and experimental results have shown that the external EM wave illuminating can be modeled as forcing terms which are added in the Telegrapher's equations 


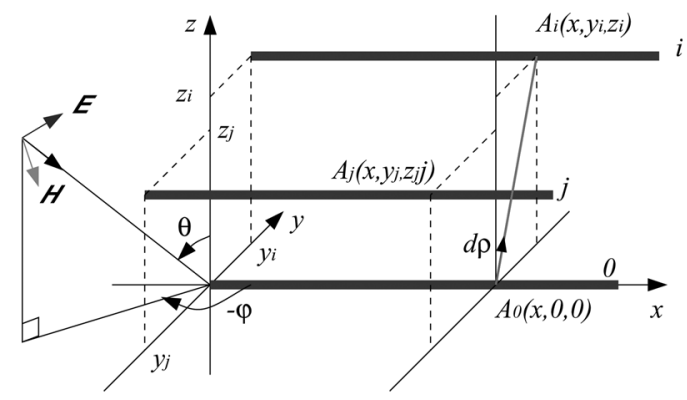

Fig. 1. MTL illuminated by EM wave.

[1], [12]-[15]. Let the illuminating EM wave be represented in the Cartesian coordinate system by

$$
\vec{E}^{\text {inc }}(s)=E_{x}^{\text {inc }}(s) \hat{a}_{x}+E_{y}^{\text {inc }}(s) \hat{a}_{y}+E_{z}^{\text {inc }}(s) \hat{a}_{z}
$$

and the TLs stretch along the $x$ direction. Assume that the reference of conductor (defined as line 0 ) is collinear with the $x$-axis (see Fig. 1). By assuming a quasi-TEM mode of propagation along an MTL consisting of $m+1$ conductors, the voltages $\mathbf{V}(x, s)$ and currents $\mathbf{I}(x, s)$ along the conductors can be represented by the $s$-domain Telegrapher's equations [1]

$$
\begin{aligned}
\frac{d}{d x} \mathbf{V}(x, s)+\mathbf{L}(x) \frac{d}{d t} \mathbf{I}(x, s)+\mathbf{R}(x) \mathbf{I}(x, s) & =\mathbf{V}_{f}(x, s) \\
\frac{d}{d x} \mathbf{I}(x, s)+\mathbf{C}(x) \frac{d}{d t} \mathbf{V}(x, s)+\mathbf{G}(x) \mathbf{V}(x, s) & =\mathbf{I}_{f}(x, s)
\end{aligned}
$$

where $\mathbf{L}(x), \mathbf{C}(x), \mathbf{R}(x)$, and $\mathbf{G}(x)$ denote the per-unit-length (PUL) inductance, capacitance, resistance, and conductance $m \times m$ matrices at point $x$, respectively. The $m$-dimensional vector forcing functions $\mathbf{V}_{f}(x, t)$ and $\mathbf{I}_{f}(x, t)$ represent the distributed voltages and distributed currents due to the incident EM wave, which are given by

$$
\begin{aligned}
& \mathbf{V}_{f}(x, s)=\left[\begin{array}{c}
\vdots \\
V_{f}^{i}(x, s) \\
\vdots
\end{array}\right]=\left[\begin{array}{c}
\vdots \\
-\frac{\partial}{\partial x} V_{t}^{i}(s)+E_{l}^{i}(s) \\
\vdots
\end{array}\right] \\
& \mathbf{I}_{f}(x, s)=\left[\begin{array}{c}
\vdots \\
I_{f}^{i}(x, s) \\
\vdots
\end{array}\right]=-(\mathbf{G}+s \mathbf{C})\left[\begin{array}{c}
\vdots \\
V_{t}^{i}(s) \\
\vdots
\end{array}\right]
\end{aligned}
$$

where $V_{f}^{i}(x, s)$ and $I_{f}^{i}(x, s)$ are the $s$-domain forcing voltage and current at point $x$ of the $i$ th line, respectively

$$
\begin{aligned}
V_{t}^{i}(x, s) & =\int_{A_{0}}^{A_{i}} \vec{E}_{t}^{\mathrm{inc}}(s) \cdot d \vec{\rho} \\
& =\int_{A_{0}}^{A_{i}}\left[E_{y}^{\text {inc }}(s) \hat{a}_{y}+E_{z}^{\text {inc }}(s) \hat{a}_{z}\right] \cdot d \vec{\rho}
\end{aligned}
$$

is the transverse voltage difference due to the transverse components of the incident electric field intensity vector $E^{i}$, where $A_{0}$ denotes the point $(x, 0,0)$, and $A_{i}$ the point $\left(x, y_{i}, z_{i}\right)$, and

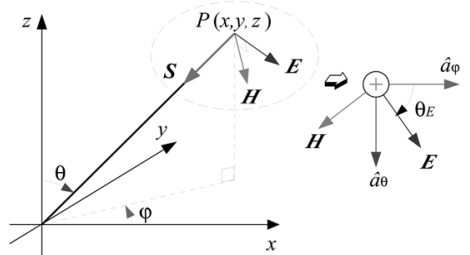

Fig. 2. Formulation of MTL illuminated by EM wave.

is the contribution of the longitudinal component of the incident electric field.

\section{B. Plane Wave}

Consider a transient electromagnetic plane wave whose propagation direction $\vec{S}$ is $-\hat{a}_{r}$ as shown in the spherical coordinate system $(r, \theta, \phi)$ (see Fig. 2). The wave propagates in free space with propagation velocity

$$
\vec{v}=-v_{0} \hat{a}_{r}=v_{x} \hat{a}_{x}+v_{y} \hat{a}_{y}+v_{z} \hat{a}_{z}
$$

where $v_{0}=\sqrt{\mu_{0} \epsilon_{0}}$. The $s$-domain representation of the wave is

$$
\vec{E}^{\text {inc }}(x, y, z, s)=\vec{e} E_{0} e^{-s \vec{k} \cdot \vec{r}}
$$

where $E_{0}$ is the waveform of the field and

$$
\vec{e}=e_{x} \hat{a}_{x}+e_{y} \hat{a}_{y}+e_{z} \hat{a}_{z}
$$

is the unit vector representing the polarization of the electric field in the Cartesian coordinate system

$$
\vec{r}=x \hat{a}_{x}+y \hat{a}_{y}+z \hat{a}_{z}
$$

is the position vector

$$
\vec{k}=-k \hat{a}_{r}=k_{x} \hat{a}_{x}+k_{y} \hat{a}_{y}+k_{z} \hat{a}_{z}
$$

is the wave vector.

The components of $\vec{e}$ along the $x-, y$-, and $z$-axes are

$$
\begin{aligned}
& e_{z}=\sin \theta_{E} \sin \theta \\
& e_{x}=-\sin \theta_{E} \cos \theta \cos \phi-\cos \theta_{E} \sin \phi \\
& e_{y}=-\sin \theta_{E} \cos \theta \sin \phi+\cos \theta_{E} \cos \phi .
\end{aligned}
$$

The components of the phase in the Cartesian coordinate system are

$$
\begin{aligned}
k_{z} & =-k \cos \theta \\
k_{x} & =-k \sin \theta \cos \phi \\
k_{y} & =-k \sin \theta \sin \phi .
\end{aligned}
$$

With the previous definitions, (9) becomes

$\vec{E}^{\text {inc }}(s)=E_{0}\left(e_{x} \hat{a}_{x}+e_{y} \hat{a}_{y}+e_{z} \hat{a}_{z}\right) e^{-s\left(k_{x} x+k_{y} y+k_{z} z\right)}$.

In the time-domain, the wave in (9) is represented by

$$
\vec{E}^{\mathrm{inc}}(x, y, z, t)=\vec{e} E_{0}\left(t-k_{x} x-k_{y} y-k_{z} z\right)
$$


where the wave components along the axes are denoted by

$$
\begin{aligned}
& k_{z}=\frac{1}{v_{z}}=-\frac{\cos \theta}{v} \\
& k_{x}=\frac{1}{v_{x}}=-\frac{\sin \theta \cos \phi}{v} \\
& k_{y}=\frac{1}{v_{y}}=-\frac{\sin \theta \sin \phi}{v} .
\end{aligned}
$$

\section{Equivalent Sources to TL With Reference Line}

By using the previous definitions, the transverse field contributions in (6) for the $i$ th conductor are derived as

$$
V_{t}^{i}(x, s)=E_{0} e^{-s k_{x} x} \rho_{i}^{+} \frac{e^{-s \tau_{i}^{+}}-1}{-s \tau_{i}^{+}}
$$

and the longitudinal field contributions in (7) for the $i$ th conductor are

$$
E_{l}^{i}(x, s)=E_{0} e^{-s k_{x} x} e_{x}\left(e^{-s \tau_{i}^{+}}-1\right)
$$

where

$$
\begin{gathered}
\tau_{i}^{+}=k_{y} y_{i}+k_{z} z_{i} \\
\rho_{i}^{+}=e_{y} y_{i}+e_{z} z_{i} .
\end{gathered}
$$

Substituting (15) and (16) into (4) and (5) results

$$
\begin{aligned}
& \mathbf{V}_{f}(x, s)=E_{0} e^{-s k_{x} x}\left[\left(e^{-s \tau_{i}^{+}}-1\right)\left(\frac{k_{x} \rho_{i}^{+}}{-\tau_{i}^{+}}+e_{x}\right)\right] \\
& \mathbf{I}_{f}(x, s)=(\mathbf{G}+s \mathbf{C}) E_{0} e^{-s k_{x} x}\left[\frac{e^{-s \tau_{i}^{+}-1}}{s \tau_{i}^{+}} \rho_{i}^{+}\right] .
\end{aligned}
$$

Inverse Laplace transform gives the time-domain counterparts of (17) and (18) as

$$
\begin{aligned}
\mathbf{V}_{f}(x, t)= & {\left[\left(\frac{k_{x} \rho_{i}^{+}}{-\tau_{i}^{+}}+e_{x}\right)\left[E_{0}\left(t-k_{x} x-\tau_{i}^{+}\right)-E_{0}\left(t-k_{x} x\right)\right]\right] } \\
\mathbf{I}_{f}(x, t)= & \mathbf{G}\left[\frac{\rho_{i}^{+}}{\tau_{i}^{+}} \int_{0}^{t}\left[E_{0}\left(\tau-k_{x} x-\tau_{i}^{+}\right)-E_{0}\left(\tau-k_{x} x\right)\right] d \tau\right] \\
& +\mathbf{C}\left[\frac{\rho_{i}^{+}}{\tau_{i}^{+}}\left[E_{0}\left(t-k_{x} x-\tau_{i}^{+}\right)-E_{0}\left(t-k_{x} x\right)\right]\right]
\end{aligned}
$$

For the quasi-TEM assumption under which the dimensions of TLs cross-sectional sizes are much smaller than the longitudinal sizes, i.e., $y_{i}, z_{i} \ll x$, we take the first two items of the following series expansion of $e^{-s \tau_{i}}$ :

$$
e^{-s \tau_{i}^{+}}=1-s \tau_{i}^{+}-\frac{\left(s \tau_{i}^{+}\right)^{2}}{2 !}-\cdots
$$

then (17) and (18) become

$$
\begin{aligned}
\mathbf{V}_{f}(x, s) & =s E_{0} e^{-s k_{x} x}\left[k_{x} \rho_{i}^{+}-e_{x} \tau_{i}^{+}\right] \\
\mathbf{I}_{f}(x, s) & =-(\mathbf{G}+s \mathbf{C}) E_{0} e^{-s k_{x} x}\left[\rho_{i}^{+}\right] .
\end{aligned}
$$

In the time-domain, (21) and (22) become

$$
\begin{aligned}
\mathbf{V}_{f}(x, t) & =\left[\left(k_{x} \rho_{i}^{+}-e_{x} \tau_{i}^{+}\right) \frac{\partial}{\partial t} E_{0}\left(t-k_{x} x\right)\right] \\
\mathbf{I}_{f}(x, t) & =-\mathbf{G}\left[\rho_{i}^{+} E_{0}\left(t-k_{x} x\right)\right]-\mathbf{C}\left[\rho_{i}^{+} \frac{\partial}{\partial t} E_{0}\left(t-k_{x} x\right)\right]
\end{aligned}
$$

\section{Equivalent Sources to TL With Infinite Ground Plane}

Assume that the reference is an infinite and perfect conductor plane located in the $x y$ plane. For an incident wave represented in (12), the reflected wave is represented by [16]

$$
\vec{E}^{\mathrm{ref}}(s)=E_{0}\left(-e_{x} \hat{a}_{x}-e_{y} \hat{a}_{y}+e_{z} \hat{a}_{z}\right) e^{-s\left(k_{x} x+k_{y} y-k_{z} z\right)} .
$$

The total fields are thus

$$
\begin{aligned}
& E_{z}^{\mathrm{tot}}(s)=E_{0} e_{z}\left(e^{-s k_{z} z}+e^{s k_{z} z}\right) e^{-s\left(k_{x} x+k_{y} y\right)} \\
& E_{x}^{\mathrm{tot}}(s)=E_{0} e_{x}\left(e^{-s k_{z} z}-e^{s k_{z} z}\right) e^{-s\left(k_{x} x+k_{y} y\right)} \\
& E_{y}^{\mathrm{tot}}(s)=E_{0} e_{y}\left(e^{-s k_{z} z}-e^{s k_{z} z}\right) e^{-s\left(k_{x} x+k_{y} y\right)} .
\end{aligned}
$$

Similarly, the transverse field contributions in (6) for the $i$ th conductor are derived in this case as

$$
V_{t}^{i}(x, s)=E_{0} e^{-s k_{x} x}\left(\rho_{i}^{+} \frac{e^{-s \tau_{i}^{+}}-1}{-s \tau_{i}^{+}}+\rho_{i}^{-} \frac{e^{s \tau_{i}^{-}}-1}{s \tau_{i}^{-}}\right)
$$

and the longitudinal field contributions in (7) for the $i$ th conductor in this case are

$$
E_{l}^{i}(s)=E_{0} e^{-s k_{x} x} e_{x}\left(e^{-s \tau_{i}^{+}}-e^{s \tau_{i}^{-}}\right)
$$

where

$$
\begin{aligned}
\tau_{i}^{-} & =k_{y} y_{i}-k_{z} z_{i} \\
\rho_{i}^{-} & =e_{y} y_{i}-e_{z} z_{i} .
\end{aligned}
$$

Substituting (27) and (28) into (4) and (5) results

$$
\begin{aligned}
\mathbf{V}_{f}(x, s)= & E_{0} e^{-s k_{x} x} \\
\times & {\left[k_{x}\left(\rho_{i}^{+} \frac{e^{-s \tau_{i}^{+}}-1}{-\tau_{i}^{+}}+\rho_{i}^{-} \frac{e^{s \tau_{i}^{-}}-1}{\tau_{i}^{-}}\right)\right.} \\
& \left.+e_{x}\left(e^{-s \tau_{i}^{+}}-e^{s \tau_{i}^{-}}\right)\right] \\
\mathbf{I}_{f}(x, s)=- & \left.(\mathbf{G}+s \mathbf{C}) \times E_{0} e^{-s k_{x} x}\right] \\
\times & {\left[\rho_{i}^{+} \frac{e^{-s \tau_{i}^{+}}-1}{-s \tau_{i}^{+}}+\rho_{i}^{-} \frac{e^{s \tau_{i}^{-}}-1}{s \tau_{i}^{-}}\right] . }
\end{aligned}
$$

In the time-domain, (29) and (30) become

$$
\begin{aligned}
\mathbf{V}_{f}(x, t)= & {\left[\left(\frac{k_{x} \rho_{i}^{+}}{-\tau_{i}^{+}}+e_{x}\right) E_{0}\left(t-k_{x} x-\tau_{i}^{+}\right)\right] } \\
& +\left[\left(\frac{k_{x} \rho_{i}^{-}}{\tau_{i}^{-}}-e_{x}\right) E_{0}\left(t-k_{x} x+\tau_{i}^{-}\right)\right] \\
& +\left[\left(\frac{k_{x} \rho_{i}^{+}}{-\tau_{i}^{+}}-\frac{k_{x} \rho_{i}^{-}}{\tau_{i}^{-}}\right) E_{0}\left(t-k_{x} x\right)\right]
\end{aligned}
$$




$$
\begin{aligned}
\mathbf{I}_{f}(x, t)= & \mathbf{G}\left[\int_{0}^{t} \frac{\rho_{i}^{+}}{\tau_{i}^{+}}\left[E_{0}\left(\tau-k_{x} x-\tau_{i}^{+}\right)-E_{0}\left(\tau-k_{x} x\right)\right] d \tau\right] \\
& -\mathbf{G}\left[\int_{0}^{t} \frac{\rho_{i}^{-}}{\tau_{i}^{-}}\left[E_{0}\left(\tau-k_{x} x+\tau_{i}^{-}\right)-E_{0}\left(\tau-k_{x} x\right)\right] d \tau\right] \\
& +\mathbf{C}\left[\frac{\rho_{i}^{+}}{\tau_{i}^{+}}\left[E_{0}\left(t-k_{x} x-\tau_{i}^{+}\right)-E_{0}\left(t-k_{x} x\right)\right]\right] \\
& -\mathbf{C}\left[\frac{\rho_{i}^{-}}{\tau_{i}^{-}}\left[E_{0}\left(t-k_{x} x+\tau_{i}^{-}\right)-E_{0}\left(t-k_{x} x\right)\right]\right] .
\end{aligned}
$$

If we take the first two items of the Taylor series expansions of $e^{-s \tau_{i}^{+}}$and $e^{s \tau_{i}^{-}},(29)$ and (30) are simplified as

$$
\begin{aligned}
\mathbf{V}_{f}(x, s) & =s E_{0} e^{-s k_{x} x} \cdot 2\left[k_{x} e_{y} y_{i}-e_{x} k_{y} y_{i}\right] \\
\mathbf{I}_{f}(x, s) & =-(\mathbf{G}+s \mathbf{C}) E_{0} e^{-s k_{x} x} \cdot 2\left[e_{y} y_{i}\right] .
\end{aligned}
$$

The time-domain counterparts of (33) and (34) are

$$
\begin{aligned}
\mathbf{V}_{f}(x, t)= & 2\left[\left(e_{y} k_{x} y_{i}-e_{x} k_{y} y_{i}\right) \frac{\partial}{\partial t} E_{0}\left(t-k_{x} x\right)\right] \\
\mathbf{I}_{f}(x, s)= & -2 \mathbf{G}\left[e_{y} y_{i} E_{0}\left(t-k_{x} x\right)\right] \\
& -2 \mathbf{C}\left[e_{y} y_{i} \frac{\partial}{\partial t} E_{0}\left(t-k_{x} x\right)\right] .
\end{aligned}
$$

\section{FDQ METHOD}

\section{A. Motivation}

The FD methods have been fully developed in the literature and are widely used to numerically solve differential equations. Consider a smooth function $f(x)$ defined on the domain $[0,1]$, which is divided by grid points $\left\{x_{i}, i=1, \ldots, n\right\}$, then the central FD framework is given by

$$
f\left(x_{i+1}\right)-f\left(x_{i-1}\right)=h \frac{d}{d x} f\left(x_{i}\right)
$$

where $h=x_{i+1}-x_{i-1}$ is the distance between two adjacent grid points. This framework is a local approximation in that the finite difference is only represented by the immediately neighboring grid points. Despite its wide popularity and uses, it requires very dense grid points and, therefore, takes computationally prohibitive time to solve large problems. The general approximation framework of Gaussian quadrature is shown as

$$
f(b)-f(a)=\int_{a}^{b} \frac{d f}{d x} d x=\sum_{i=1}^{n} c_{i} \frac{d}{d x} f\left(x_{i}\right)
$$

where $c_{i}$ 's are the coefficients which are determined by the orthogonal polynomials in the particular Gaussian rules. Compared to the FD framework in (37), the Gaussian Quadrature in (38) is a global approximation in that the difference between $u(a)$ and $u(b)$ is represented by all the grid points over the entire domain.

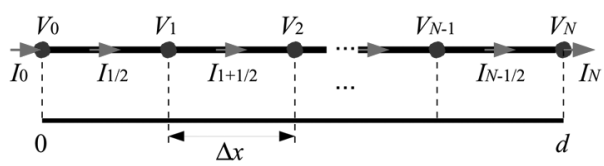

Fig. 3. FDQ framework.

The proposed method is to integrate the finite difference and quadrature methods, the general framework of which is shown by

$$
f\left(x_{i+1}\right)-f\left(x_{i}\right)=\sum_{k=1}^{n} c_{i k} w_{i k} \frac{d}{d x} f\left(x_{k}\right)
$$

where $w_{i j}$ 's are the weighted coefficients. The right-hand side representation is apparently a global quadrature approximation, which is the same as that in (38); however, it is to compute the finite difference at the left-hand side. The $F D Q$ coefficients $c_{k}$ 's are determined by using testing function approach, similarly to the Galerkin's method.

Once the positions of grid points are fixed, the corresponding FDQ coefficients are completely determined, which are fixed constants, no matter in what applications the differential equations appear. In general, the set of grid points are selected carefully so that they are symmetric with respect to the center of the domain; alternatively, they can be equally spacing points over the domain. Next, we develop the FDQ method by showing the approximation scheme and applying it to a simple transmission line with EM wave coupling.

\section{B. FDQ Modeling by an Example}

For simplicity, the FDQ modeling approach is first developed on a simple example of a uniform two-wire TL with one of the wires being the reference. A direct numerical technique, FDQ methods do not need to decouple the MTL, therefore, its application to nonuniform and/or multiconductor TL is straightforward extended. Assume that a TL stretches from 0 to $d$ along the $x$-axis of a Cartesian coordinate system, where $d$ is the length of the line. With $V(x, s)$ and $I(x, s)$ being, respectively, the Laplace-domain voltage and current vectors at point $x$, the normalized Telegrapher's equations in $s$-domain can be written as

$$
\begin{aligned}
\frac{d}{d x} V(x, s) & =-(s L+R) I(x, s)+V_{f}(x, s) \\
\frac{d}{d x} I(x, s) & =-(s C+G) V(x, s)+I_{f}(x, s)
\end{aligned}
$$

where $R(x), L(x), G(x)$, and $C(x)$ are the per-unit-length (PUL) parameters, representing resistance, inductance, conductance, and capacitance parameters, respectively.

As shown in Fig. 3, we choose to equally discretize the transmission line to $N$ small sections, each of which has the length of $\Delta x=d / N$. There are two sets of grid points: one set are at integer-spatial positions as $x_{i}=i \Delta x, i=0, \ldots, N$, the other set are those at half-spatial positions as $x_{i+1 / 2}=(i+1 / 2) \Delta x$, $i=0, \ldots,(N-1)$. The grid points are numbered in such a way that the voltages are evaluated at integer-spatial positions as $V_{i}=V\left(x_{i}, s\right), i=0, \ldots, N$, and the currents are evaluated at half-spatial positions as $I_{i+1 / 2}=I\left(x_{i+1 / 2}, s\right)$. In addi- 
tion, the currents $I_{0}=I(0, s)$ and $I_{N}=I(d, s)$ are input and output currents at the port, respectively. For clarity of the intermediate use in the context, we define the intermediate voltages $V_{i+1 / 2}=V\left(x_{i+1 / 2}, s\right), i=0, \ldots,(N-1)$ and the intermediate currents $I_{i}=I\left(x_{i}, s\right), i=1, \ldots,(N-1)$.

For the finite difference of voltage, the FDQ approximation framework is

$$
V_{i+1}-V_{i}=\Delta x \sum_{k=0}^{N-1} a_{i k} \frac{d}{d x} V_{k+1 / 2}, \quad i=0, \ldots,(N-1) .
$$

For the finite difference of voltage, the FDQ approximation framework is

$$
I_{i+1 / 2}-I_{i-1 / 2}=\Delta x \sum_{k=0}^{N} b_{i k} \frac{d}{d x} I_{k}, \quad i=1, \ldots,(N-1)
$$

for internal grid points and

$$
\begin{aligned}
I_{1 / 2}-I_{0} & =\Delta x \sum_{k=0}^{N} b_{0 k} \frac{d}{d x} I_{k} \\
I_{N}-I_{N-1 / 2} & =\Delta x \sum_{k=0}^{N} b_{N k} \frac{d}{d x} I_{k}
\end{aligned}
$$

for the left and right boundary schemes, respectively.

Substituting (40) and (41) into (42)-(45), we obtain

$$
\begin{aligned}
& V_{i+1}-V_{i}=\Delta x \sum_{k=0}^{N-1} a_{i k} \\
& \times\left[(s L+R) I_{k+1 / 2}+V_{f}\left(x_{k+1 / 2}, s\right)\right], \\
& i=0, \ldots,(N-1) \\
& I_{i+1 / 2}-I_{i-1 / 2}=\Delta x \sum_{k=0}^{N} b_{i k}\left[(s C+G) V_{k}+I_{f}\left(x_{k}, s\right)\right] \text {, } \\
& i=1, \ldots,(N-1)
\end{aligned}
$$

for the internal grid points and

$$
\begin{aligned}
I_{1 / 2}-I_{0} & =\Delta x \sum_{k=0}^{N} b_{0 k}\left[(s C+G) V_{k}+I_{f}\left(x_{k}, s\right)\right] \\
I_{N}-I_{N-1 / 2} & =\Delta x \sum_{k=0}^{N} b_{N k}\left[(s C+G) V_{k}+I_{f}\left(x_{k}, s\right)\right]
\end{aligned}
$$

for the boundary points.

Equations (46)-(49) are the FDQ approximation framework of the two-wire TL in $s$-domain. The weighting coefficients $a_{i j}$, $i, j=0, \ldots,(N-1)$ and $b_{i j}, i, j=0, \ldots, N$ are determined by using the weighting residual method, a test-function-based approach similar to Galerkin's method is employed. Consider the following function set:

$$
1, x, x^{2}, \ldots, x^{n}, \ldots
$$

defined in the domain $[0,1]$. In order for (42) to be exact in the $N$-dimensional subspace, every item in the function set $x^{n}, n=$
$0, \ldots, N$ serves as a test function to fit (42). Substituting every function $x^{n}$ into (42), and noting that in this case $V_{i}=\left[x^{n}\right]_{x=x_{i}}$, $V_{k+1 / 2}=\left[x^{n}\right]_{x=x_{k+1 / 2}}$, it follows:

$$
x_{i+1}^{n}-x_{i}^{n}=\sum_{k=0}^{N-1} a_{i k} n x_{k+1 / 2}^{n-1} .
$$

It is observed that the $N$ in (51) $(n=0, \ldots, N)$ constitute a Vandermonde matrix equation for a given $i$, with $a_{i k}$ 's being the unknowns. Due to the property of Vandermonde matrix equation, the coefficient $a_{i 0}, a_{i 1}, \ldots, a_{i(N-1)}$ are uniquely determined by solving the previous equations. Repeatedly applying the same process to $i=0, \ldots,(N-1)$, an $N \times N$ matrix of coefficients is obtained

$$
\mathbf{A}=\left[\begin{array}{cccc}
a_{00} & a_{01} & \ldots & a_{0(N-1)} \\
\vdots & \vdots & \ddots & \vdots \\
a_{(N-1) 0} & a_{(N-1) 1} & \ldots & a_{(N-1)(N-1)}
\end{array}\right]
$$

According to Galerkin's method, the approximation scheme in (51) has an accuracy $O\left(\Delta x^{N+1}\right)$, where $\Delta x$ is the mesh size between two grid points.

Similarly, substituting the first $(N+2)$ functions of $x^{n}$ into (43)-(45) results the Vandermonde matrix equation $(n=0, \ldots, N)$

$$
x_{i+1 / 2}^{n}-x_{i-1 / 2}^{n}=\sum_{k=0}^{N} b_{i k} n x_{k}^{n-1} .
$$

Repeatedly solving the previous Vandermonde matrix equation for $i=0, \ldots, N$ leads to an $(N+1) \times(N+1)$ matrix of coefficients

$$
\mathbf{B}=\left[\begin{array}{cccc}
b_{00} & b_{01} & \ldots & b_{0 N} \\
\vdots & \vdots & \ddots & \vdots \\
b_{N 0} & b_{N 1} & \ldots & b_{N N}
\end{array}\right]
$$

The approximation scheme in (53) has an accuracy $O\left(\Delta x^{N+2}\right)$.

Once the positions of the grid points are fixed, the previous testing-function approach leads to constant FDQ coefficient matrices, no matter in what applications the TL equations appear. With the precalculated coefficient matrices, (46)-(49) are rewritten as

$$
\left[\begin{array}{cc}
\mathbf{B} Y(s) & \mathbf{P}^{T} \\
\mathbf{P} & \mathbf{A} Z(s)
\end{array}\right]\left[\begin{array}{l}
\mathbf{V} \\
\mathbf{I}
\end{array}\right]=\left[\begin{array}{ll}
\mathbf{0} & \mathbf{B} \\
\mathbf{A} & \mathbf{0}
\end{array}\right]\left[\begin{array}{l}
\mathbf{V}_{f} \\
\mathbf{I}_{f}
\end{array}\right]+\left[\begin{array}{l}
\mathbf{b} \\
\mathbf{0}
\end{array}\right]\left[\begin{array}{c}
I_{0} \\
I_{N}
\end{array}\right]
$$

where

$$
\begin{aligned}
Z(s) & =s L+R \\
Y(s) & =s C+G \\
\mathbf{V} & =\left[\begin{array}{llll}
V_{0} & V_{1} & \cdots & V_{N}
\end{array}\right]^{T} \\
\mathbf{I} & =\left[\begin{array}{llll}
I_{1 / 2} & I_{1+1 / 2} & \cdots & I_{N-1 / 2}
\end{array}\right]^{T} \\
\mathbf{V}_{f} & =\left[\begin{array}{llll}
V_{f}\left(x_{1 / 2}\right) & V_{f}\left(x_{1+1 / 2}\right) & \cdots & V_{f}\left(x_{N-1 / 2}\right)
\end{array}\right]^{T} \\
\mathbf{I}_{f} & =\left[\begin{array}{llll}
I_{f}\left(x_{0}\right) & I_{f}\left(x_{1}\right) & \cdots & I_{f}\left(x_{N}\right)
\end{array}\right]^{T}
\end{aligned}
$$


where $\mathbf{P}$ is an $N \times(N+1)$ matrix

$$
\mathbf{P}=\left[\begin{array}{ccccc}
-1 & 1 & & & \\
& -1 & 1 & & \\
& & \ddots & \ddots & \\
& & & -1 & 1
\end{array}\right]
$$

$\mathbf{P}^{T}$ is the transpose matrix of $\mathbf{P}$, and $\mathbf{b}$ is an $(N+1) \times 2$ connecting matrix of the external exiting current sources

$$
\mathbf{b}=\left[\begin{array}{ccccc}
1 & 0 & \ldots & 0 & 0 \\
0 & 0 & \ldots & 0 & -1
\end{array}\right]^{T}
$$

Note that $I_{0}$ and $I_{N}$ are the external exciting current sources.

\section{General FDQ Approximation Framework}

As shown, both $A$ and $B$ in (52) and (54) are dense matrices. If their ranks are large, solving (55) will be computationally expensive. Nonetheless, if $N$ is large, the closed-form formula to compute $a_{i j}$ (or $b_{i j}$ ) in the Vandermonde matrix equation formed by (51) [or (53)] will not be available, and the condition number of the Vandermonde matrix equation will be very large and therefore ill-conditioned. As a result, it is suggested that the previous approach with the dense matrices $A$ and $B$ are only applicable to small scale problems, where $N$ is no more than 12 and the electrical length of transmission line is less than 3 wavelengths.

In order to apply the FDQ technique to large scale problem where the electrical size could be as large as 40 wavelengths, we modify the original FDQ method such that it takes the scheme as shown before at the boundary grid points while it adopts the following central FDQ scheme at other grid points.

Shown in Fig. 4, associated with function $f(x)$ defined on the domain there are two types of uniformly spaced grid points: those on integer grid points $(x=i \Delta x)$ and those on half integer gird points $(x=(i+1 / 2) \Delta x)$. Note that in Fig. 4 the subscripts of $f$ index the position of function values, e.g., $f_{i+1 / 2}=$ $f\left(x_{i+1 / 2}\right)$ and $f_{i}=f\left(x_{i}\right)$. We first show how to derive the central FDQ framework for the finite difference at half integer grid points, i.e., $\left(f_{i+1 / 2}-f_{i-1 / 2}\right)$. Suppose a sliding window centers at the $i$ th grid point and holds $n$ grid points, where $n$ is an odd number because the $n$ grid points are symmetrically distributed with respect to the $i$ th grid point. That means, the sliding window spans from the $(i-n / 2)$ th point to $(i+n / 2)$ th point. The central FDQ framework at half integer grid points follows:

$$
f_{i+1 / 2}-f_{i-1 / 2}=\Delta x \sum_{j=-(n-1) / 2}^{(n-1) / 2} c_{j} \frac{d}{d x} f_{i+j}
$$

where the coefficients from $c_{-(n-1) / 2}$ to $c_{(n-1) / 2}$ are determined by using the test functions in (50) and solving the Vandemonde matrix equations as shown in Section III-B. In order to determine the $n$ coefficients, test functions up to $x^{n}$ in (50) are needed; therefore, the approximation framework has accuracy order $O\left(\Delta x^{n+1}\right)$. It is easy to verify that the scheme is symmetric with respect to $f_{i}$, i.e., $c_{-(n-1) / 2}=c_{(n-1) / 2}$ and so on.

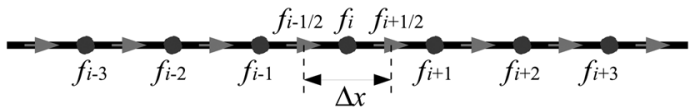

Fig. 4. FDQ central framework.

Depending on different widths of the sliding window in Fig. 4, the FDQ approximation schemes in (56) and (62) have different approximation orders. In the following, the central FDQ approximation scheme with $n$th order is denoted as FDQn.

The simplest case is $n=1$, which reduces (56) to

$$
f_{i+1 / 2}-f_{i-1 / 2}=\Delta x \frac{d}{d x} f_{i}
$$

Apparently, this scheme is that of the central finite difference having accuracy order $O\left(\Delta x^{2}\right)$ (FDQ2 or FD2).

If $n=3$, (56) becomes

$$
\begin{aligned}
f_{i+1 / 2}- & f_{i-1 / 2} \\
& =\Delta x\left(\frac{1}{24} \frac{d}{d x} f_{i-1}+\frac{11}{12} \frac{d}{d x} f_{i}+\frac{1}{24} \frac{d}{d x} f_{i+1}\right) .
\end{aligned}
$$

This scheme has accuracy order $O\left(\Delta x^{4}\right)$ (FDQ4). Actually, this approximation scheme is exactly the Ty operator in the literature [17].

If $n=5$ and $n=7$, respectively, (56) becomes, respectively, (FDQ6)

$$
\begin{aligned}
f_{i+1 / 2}-f_{i-1 / 2}=\Delta x & -\frac{17}{5760} \frac{d}{d x} f_{i-2}+\frac{77}{1440} \frac{d}{d x} f_{i-1} \\
& +\frac{863}{960} \frac{d}{d x} f_{i}+\frac{77}{1440} \frac{d}{d x} f_{i+1} \\
& \left.-\frac{17}{5760} \frac{d}{d x} f_{i+2}\right)
\end{aligned}
$$

and (FDQ8)

$$
\begin{gathered}
f_{i+1 / 2}-f_{i-1 / 2} \\
=\Delta x\left(\frac{367}{967680} \frac{d}{d x} f_{i-3}-\frac{281}{53760} \frac{d}{d x} f_{i-2}\right. \\
+\frac{6361}{107520} \frac{d}{d x} f_{i-1}+\frac{215641}{241920} \frac{d}{d x} f_{i} \\
+\frac{6361}{107520} \frac{d}{d x} f_{i+1}-\frac{281}{53760} \frac{d}{d x} f_{i+2} \\
\left.+\frac{367}{967680} \frac{d}{d x} f_{i+3}\right) .
\end{gathered}
$$

Similarly, higher order FDQ schemes can be developed. However, as higher order FDQ schemes span wider in the $x$ direction and, therefore, cover more grid points, the application may become inconvenient.

To evaluate the numerical dispersion of the approximation schemes, next, we show the Fourier analysis. Assume that $f$ is 


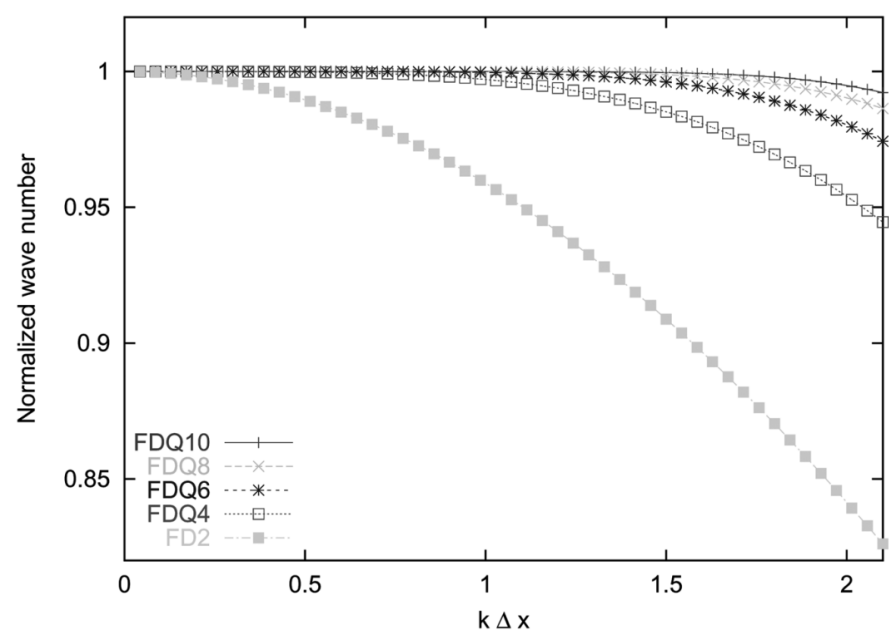

Fig. 5. Numerical dispersion of FDQ central framework.

TABLE I

NUMBER OF CELLS PER WAVELENGTH FOR DIFFERENT FDQ SCHEMES

\begin{tabular}{|l||c|c|c|c|c|}
\hline Error (\%) & FD2 & FDQ4 & FDQ6 & FDQ8 & FDQ10 \\
\hline 1 & 12.8 & 4.6 & 3.5 & 3.1 & 2.9 \\
\hline 0.1 & 40.8 & 8.2 & 5.3 & 4.3 & 3.8 \\
\hline
\end{tabular}

of the form of $e^{-j(\omega t-k x)}$, which describes a plane wave propagating in the $+x$ direction. If we plug the wave representation into (57), it follows:

$$
k=\sin \frac{\left(\frac{k \Delta x}{2}\right)}{\left(\frac{\Delta x}{2}\right)} .
$$

The LHS is the wave number of the plane wave, while the RHS is the wave number of the approximation scheme. The normalized wave number of the FD2 scheme is, therefore, $\sin (k \Delta x / 2) /(k \Delta x / 2)$. Similarly, the normalized wave numbers of the FDQ4, FDQ6, and FDQ8 schemes can be derived. Fig. 5 shows the comparison of the normalized wave numbers versus $k \Delta x$ for the approximation schemes.

Obviously, FD2 scheme has very limited spectral bandwidth when compared to other FDQ schemes, and higher FDQ schemes have wider spectral bandwidth. Based on the normalized wave number we can observe the accuracy of the approximation schemes corresponding to the density of the grid points. Note that $k \Delta x=2 \pi / C P W$, where $C P W=\lambda / \Delta x$ is the number of cells per wavelength. Table I shows the required CPWs at the phase errors of $1 \%$ and $0.1 \%$, respectively.

It is demonstrated that, with the order of the FDQ scheme going to higher, the resolution it needed approaches the Nyquist limit CPW of 2.

Similarly, the FDQ approximation scheme at integer grid points can be derived

$$
f_{i+1}-f_{i}=\Delta x \sum_{j=-(n-1) / 2}^{(n-1) / 2} c_{j} \frac{d}{d x} f_{i+j+1 / 2} .
$$

Mathematical analysis finds that the FDQ schemes at integergrid points [see (62)] have the same coefficients as those at halfgrid points [see (56)], consequently, (62) has the same numerical dispersion as their counterpart (56).
Given a required accuracy, an $n$th order FDQ central scheme and its required number of CPWs can be selected according to Table I for large scale transmission line problems. The central schemes in (62) and (56) can then be applied to transmission line modeling at the central grid points where the approximation frameworks can fit. At the boundary points where the frameworks do not fit, the $n$th order FDQ frameworks are determined following the approach described in Section III-B. As a result, we obtain a matrix equation that has the same form as (55), except that matrices $\mathbf{A}$ and $\mathbf{B}$ in this case are as follows:

$\mathbf{A}=\left[\begin{array}{ccccc}a_{00} & \cdots & a_{0(n-1)} & & \\ \vdots & & \vdots & & \\ c_{(n-1) / 2} & \ldots & c_{(n+1) / 2} & & \\ & \ddots & & \ddots & \\ & & c_{(n-1) / 2} & \ldots & c_{(n+1) / 2} \\ & & \vdots & & \vdots \\ & & a_{(N-1)(N-n+1)} & \ldots & a_{(N-1)(N-1)}\end{array}\right]$

$$
\mathbf{B}=\left[\begin{array}{ccccc}
b_{00} & \cdots & b_{0(n-1)} & & \\
\vdots & & \vdots & & \\
c_{(n-1) / 2} & \cdots & c_{(n+1) / 2} & & \\
& \ddots & & \ddots & \\
& & c_{(n-1) / 2} & \ldots & c_{(n+1) / 2} \\
& & \vdots & & \vdots \\
& & b_{N(N-n+1)} & \ldots & b_{N N}
\end{array}\right]
$$

It is shown that each row of matrices $\mathbf{A}$ and $\mathbf{B}$ has $n$ entries located around the diagonal.

\section{FDQ MOdeling OF NONUNIFORM TLS}

In order to concisely express some of the manipulations required in a matrix, the following terminologies are defined here at first.

For an $m \times m$ function matrix $\mathbf{F}(x)$ whose entries are functions $f_{i j}(x), i, j=1, \ldots, m$, defined on the domain $x \in[0, d]$, the integer grid points are $x_{i}=i \Delta x, i=0, \ldots, N$, and the half grid points are $x_{i+1 / 2}=(i+1 / 2) \Delta x, i=0, \ldots,(N-1)$, where $\Delta x=d / N$.

Definition 1: An integer-grid-point discretization operator $\mathbf{D}_{I}$ discretizes $\mathbf{F}(x)$ into an $(N+1) m \times(N+1) m$ block diagonal matrix, with the blocks being the matrices $\mathbf{F}(x)$ defined at the grid points $\left\{x_{0}, x_{1}, \ldots, x_{N}\right\}$, respectively

$$
\mathbf{D}_{I}(\mathbf{F})=\operatorname{diag}\left\{\mathbf{F}\left(x_{0}\right), \mathbf{F}\left(x_{1}\right), \ldots, \mathbf{F}\left(x_{N}\right)\right\} .
$$

Definition 2: In the special case of $m=1$ in Definition 1, an integer-grid-point discretization operator $\mathbf{D}_{I}$ discretizes a function $f(x)$ into an $(N+1) \times(N+1)$ diagonal matrix

$$
\mathbf{D}_{I}(f)=\operatorname{diag}\left\{f\left(x_{0}\right), f\left(x_{1}\right), \ldots, f\left(x_{N}\right)\right\} .
$$

Definition 3: A half-grid-point discretization operator $\mathbf{D}_{H}$ discretizes $\mathbf{F}(x)$ into an $N m \times N m$ block diagonal matrix, with the blocks being the matrices $\mathbf{F}(x)$ defined at the grid points $\left\{x_{1 / 2}, x_{1+1 / 2}, \ldots, x_{N-1 / 2}\right\}$, respectively

$$
\mathbf{D}_{H}(\mathbf{F})=\operatorname{diag}\left\{\mathbf{F}\left(x_{1 / 2}\right), \mathbf{F}\left(x_{1+1 / 2}\right), \ldots, \mathbf{F}\left(x_{N-1 / 2}\right)\right\} .
$$



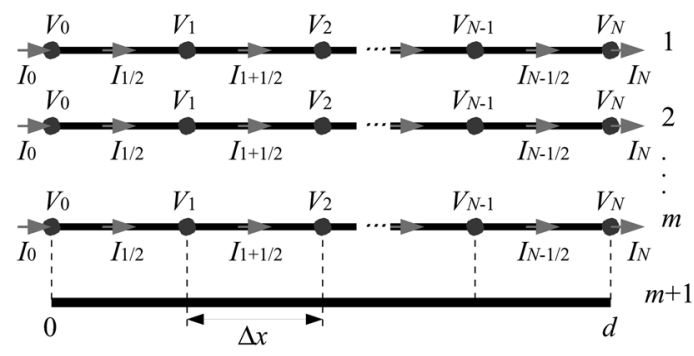

Fig. 6. FDQ framework of MTL.

Definition 4: In the special case of $m=1$ in Definition 3, a half-grid-point discretization operator $\mathbf{D}_{H}$ discretizes a function $f(x)$ into an $N \times N$ diagonal matrix

$$
\mathbf{D}_{H}(f)=\operatorname{diag}\left\{f\left(x_{1 / 2}\right), f\left(x_{1+1 / 2}\right), \ldots, f\left(x_{N-1 / 2}\right)\right\} \text {. }
$$

Definition 5: Given two matrices $\mathbf{X}=\left[x_{i j}\right], i=1, \ldots, m$, $j=1, \ldots, n$ and $\mathbf{Y}=\left[y_{i j}\right], i=1, \ldots, p, j=1, \ldots, q$, the Kronecker Product $\mathbf{X} \otimes \mathbf{Y}$ is an $m p \times n q$ matrix [18]

$$
\mathbf{X} \otimes \mathbf{Y}=\left[\begin{array}{cccc}
x_{11} \mathbf{Y} & x_{12} \mathbf{Y} & \ldots & x_{1 n} \mathbf{Y} \\
x_{21} \mathbf{Y} & x_{22} \mathbf{Y} & \ldots & x_{2 n} \mathbf{Y} \\
\vdots & \vdots & \ddots & \vdots \\
x_{m 1} \mathbf{Y} & x_{m 2} \mathbf{Y} & \ldots & x_{m n} \mathbf{Y}
\end{array}\right]
$$

\section{A. Nonuniform Two-Wire TL}

Based on (55), the frequency-domain Telegrapher's equations of a two-wire transmission line are straightforward transformed into

$$
\begin{aligned}
& {\left[\begin{array}{cc}
\mathbf{B} \cdot \mathbf{D}_{I}(G) & \mathbf{P}^{T} \\
\mathbf{P} & \mathbf{A} \cdot \mathbf{D}_{H}(R)
\end{array}\right]\left[\begin{array}{l}
\mathbf{V} \\
\mathbf{I}
\end{array}\right]} \\
& +s\left[\begin{array}{cc}
\mathbf{B} \cdot \mathbf{D}_{I}(C) & \mathbf{0} \\
\mathbf{0} & \mathbf{A} \cdot \mathbf{D}_{H}(L)
\end{array}\right]\left[\begin{array}{l}
\mathbf{V} \\
\mathbf{I}
\end{array}\right] \\
& \quad=\left[\begin{array}{cc}
\mathbf{0} & \mathbf{B} \\
\mathbf{A} & \mathbf{0}
\end{array}\right]\left[\begin{array}{l}
\mathbf{V}_{f} \\
\mathbf{I}_{f}
\end{array}\right]+\left[\begin{array}{l}
\mathbf{b} \\
\mathbf{0}
\end{array}\right]\left[\begin{array}{l}
I_{0} \\
I_{N}
\end{array}\right]
\end{aligned}
$$

where the variables and matrices are defined as in (55), except that the following matrices are defined by (66) and (68):

$$
\begin{aligned}
\mathbf{D}_{H}(L) & =\operatorname{diag}\left\{L\left(x_{1 / 2}\right), L\left(x_{1+1 / 2}\right), \ldots, L\left(x_{N-1 / 2}\right)\right\} \\
\mathbf{D}_{H}(R) & =\operatorname{diag}\left\{R\left(x_{1 / 2}\right), R\left(x_{1+1 / 2}\right), \ldots, R\left(x_{N-1 / 2}\right\}\right. \\
\mathbf{D}_{I}(C) & =\operatorname{diag}\left\{C\left(x_{0}\right), C\left(x_{1}\right), \ldots, C\left(x_{N}\right\}\right. \\
\mathbf{D}_{I}(G) & =\operatorname{diag}\left\{G\left(x_{0}\right), G\left(x_{1}\right), \ldots, G\left(x_{N}\right)\right\} .
\end{aligned}
$$

\section{B. Nonuniform MTLs}

The governing equations of a general $(m+1)$ MTL (see Fig. 6) are shown in (2) and (3), where $\mathbf{V}(x, s)$ and $\mathbf{I}(x, s)$ represent the distributed $m$-dimensional voltage vector and current vector, respectively

$$
\begin{aligned}
\mathbf{V}(x) & =\left[\begin{array}{lllll}
V^{1}(x, s) & \cdots & V^{i}(x, s) & \cdots & V^{m}(x, s)
\end{array}\right]^{T} \\
\mathbf{I}(x) & =\left[\begin{array}{lllll}
I^{1}(x, s) & \cdots & I^{i}(x, s) & \cdots & I^{m}(x, s)
\end{array}\right]^{T}
\end{aligned}
$$

where $V^{i}(x, s)$ and $I^{i}(x, s)$ are the distributed voltage and current at the $i$ th line of the $(m+1)$ MTL. The PUL parameters $\mathbf{R}(x), \mathbf{L}(x), \mathbf{C}(x)$, and $\mathbf{G}(x)$ are $(m \times m)$ matrices

$$
\begin{aligned}
\mathbf{R}(x) & =\left[\begin{array}{ccc}
R^{11}(x) & \ldots & R^{1 m}(x) \\
\vdots & \ddots & \vdots \\
R^{m 1}(x) & \ldots & R^{m m}(x)
\end{array}\right] \\
\mathbf{L}(x) & =\left[\begin{array}{ccc}
L^{11}(x) & \ldots & L^{1 m}(x) \\
\vdots & \ddots & \vdots \\
L^{m 1}(x) & \ldots & L^{m m}(x)
\end{array}\right] \\
\mathbf{C}(x) & =\left[\begin{array}{ccc}
C^{11}(x) & \ldots & C^{1 m}(x) \\
\vdots & \ddots & \vdots \\
C^{m 1}(x) & \ldots & C^{m m}(x)
\end{array}\right] \\
\mathbf{G}(x) & =\left[\begin{array}{ccc}
G^{11}(x) & \ldots & G^{1 m}(x) \\
\vdots & \ddots & \vdots \\
G^{m 1}(x) & \ldots & G^{m m}(x)
\end{array}\right] .
\end{aligned}
$$

Using Definition 5, (70) can be straightforward extended to the MTLs

$$
\begin{aligned}
& {\left[\begin{array}{cc}
\mathbf{B} \otimes \mathbf{D}_{I}(\mathbf{G}) & {[\mathbf{P} \otimes \mathbf{U}]^{T}} \\
\mathbf{P} \otimes \mathbf{U} & \mathbf{A} \otimes \mathbf{D}_{H}(\mathbf{R})
\end{array}\right]\left[\begin{array}{c}
\widehat{\mathbf{V}} \\
\widehat{\mathbf{I}}
\end{array}\right]} \\
& +s\left[\begin{array}{cc}
\mathbf{B} \otimes \mathbf{D}_{I}(\mathbf{C}) & \mathbf{0} \\
\mathbf{0} & \mathbf{A} \otimes \mathbf{D}_{H}(\mathbf{L})
\end{array}\right]\left[\begin{array}{c}
\widehat{\mathbf{V}} \\
\widehat{\mathbf{I}}
\end{array}\right] \\
& \quad=\left[\begin{array}{cc}
\mathbf{0} & \mathbf{B} \otimes \mathbf{U} \\
\mathbf{A} \otimes \mathbf{U} & \mathbf{0}
\end{array}\right]\left[\begin{array}{c}
\widehat{\mathbf{V}}_{f} \\
\widehat{\mathbf{I}}_{f}
\end{array}\right]+\left[\begin{array}{c}
\mathbf{b} \otimes \mathbf{U} \\
\mathbf{0}
\end{array}\right]\left[\begin{array}{c}
\mathbf{I}_{0} \\
\mathbf{I}_{N}
\end{array}\right]
\end{aligned}
$$

where $\mathbf{U}$ is an $m \times m$ unit matrix, $\mathbf{D}_{H}(\mathbf{R}), \mathbf{D}_{H}(\mathbf{L}), \mathbf{D}_{I}(\mathbf{C})$, $\mathbf{D}_{I}(\mathbf{G})$ are defined as in (65) and (67)

$$
\begin{aligned}
\mathbf{D}_{H}(\mathbf{L}) & =\operatorname{diag}\left\{\mathbf{L}\left(x_{1 / 2}\right), \mathbf{L}\left(x_{1+1 / 2}\right), \ldots, \mathbf{L}\left(x_{N-1 / 2}\right)\right\} \\
\mathbf{D}_{H}(\mathbf{R}) & =\operatorname{diag}\left\{\mathbf{R}\left(x_{1 / 2}\right), \mathbf{R}\left(x_{1+1 / 2}\right), \ldots, \mathbf{R}\left(x_{N-1 / 2}\right\}\right. \\
\mathbf{D}_{I}(\mathbf{C}) & =\operatorname{diag}\left\{\mathbf{C}\left(x_{0}\right), \mathbf{C}\left(x_{1}\right), \ldots, \mathbf{C}\left(x_{N}\right\}\right. \\
\mathbf{D}_{I}(\mathbf{G}) & =\operatorname{diag}\left\{\mathbf{G}\left(x_{0}\right), \mathbf{G}\left(x_{1}\right), \ldots, \mathbf{G}\left(x_{N}\right)\right\}
\end{aligned}
$$

where $\widehat{\mathbf{V}}$ and $\widehat{\mathbf{I}}$ are $m N$ - dimensional vectors

$$
\begin{aligned}
\widehat{\mathbf{V}} & =\left[\begin{array}{lllll}
\mathbf{V}_{0} & \cdots & \mathbf{V}_{i} & \cdots & \mathbf{V}_{N}
\end{array}\right]^{T} \\
\widehat{\mathbf{I}} & =\left[\begin{array}{lllll}
\mathbf{I}_{1 / 2} & \cdots & \mathbf{I}_{i+1 / 2} & \cdots & \mathbf{I}_{N-1 / 2}
\end{array}\right]^{T}
\end{aligned}
$$

where

$$
\begin{aligned}
\mathbf{V}_{i} & =\left[\begin{array}{lll}
V_{i}^{1} & \cdots & V_{i}^{m}
\end{array}\right]=\left[\begin{array}{lll}
V^{1}\left(x_{i}\right) & \cdots & V^{m}\left(x_{i}\right)
\end{array}\right] \\
\mathbf{I}_{i+1 / 2} & =\left[\begin{array}{lll}
I_{i+1 / 2}^{1} & \cdots & I_{i+1 / 2}^{m}
\end{array}\right] \\
& =\left[\begin{array}{lll}
I^{1}\left(x_{i+1 / 2}\right) & \cdots & I^{m}\left(x_{i+1 / 2}\right)
\end{array}\right]
\end{aligned}
$$

where $\widehat{\mathbf{V}}_{f}$ and $\widehat{\mathbf{I}}_{f}$ are $(N-1) m$-dimensional and $N m$-dimensional vectors, respectively

$$
\begin{aligned}
\widehat{\mathbf{V}}_{f} & =\left[\mathbf{V}_{f}\left(x_{1 / 2}\right) \cdots \mathbf{V}_{f}\left(x_{i+1 / 2}\right) \cdots \mathbf{V}_{f}\left(x_{N-1 / 2}\right)\right]^{T} \\
\widehat{\mathbf{I}}_{f} & =\left[\mathbf{I}_{f}\left(x_{0}\right) \ldots \mathbf{I}_{f}\left(x_{i}\right) \cdots \mathbf{I}_{f}\left(x_{N}\right)\right]^{T}
\end{aligned}
$$

where

$$
\begin{aligned}
\mathbf{V}_{f}\left(x_{i+1 / 2}\right) & =\left[V_{f}^{1}\left(x_{i+1 / 2}\right) \cdots V_{f}^{m}\left(x_{i+1 / 2}\right)\right] \\
\mathbf{I}_{f}\left(x_{i}\right) & =\left[I_{f}^{1}\left(x_{i}\right) \cdots I_{f}^{m}\left(x_{i}\right)\right]
\end{aligned}
$$




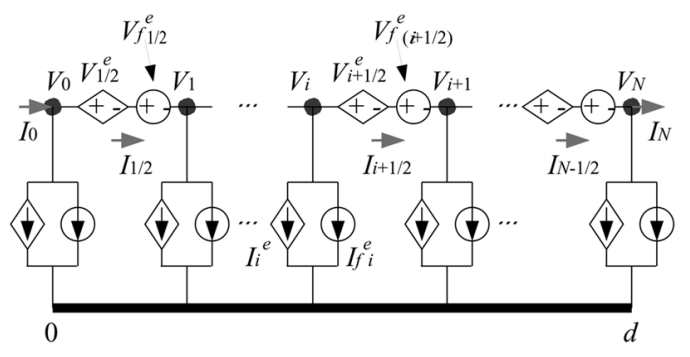

Fig. 7. Equivalent circuit of two-wire TL.

and $\mathbf{I}_{0}$ and $\mathbf{I}_{N}$ are $m$-dimensional vectors

$$
\begin{aligned}
\mathbf{I}_{0} & =\left[\begin{array}{lllll}
I_{0}^{1} & \cdots & I_{0}^{i} & \cdots & I_{0}^{m}
\end{array}\right]^{T} \\
\mathbf{I}_{N} & =\left[\begin{array}{lllll}
I_{N}^{1} & \cdots & I_{N}^{i} & \cdots & I_{N}^{m}
\end{array}\right]^{T} .
\end{aligned}
$$

Inverse Laplace transforms of (70) and (71) lead to first-order ordinary differential equations, which represent the time domain models of interconnects. The time domain responses at the ends of transmission lines can be obtained by solving ordinary differential equations.

\section{Equivalent Circuit Models}

The frequency-domain equations derived earlier can be transformed into the time domain. On the other hand, a model practically offers more flexibility if it can be integrated with simulators like SPICE. In order for the FDQ-based models to be incorporated into SPICE simulators, the equivalent circuit models are derived.

In (70), if we define

$$
\begin{aligned}
\mathbf{V}^{e} & =\mathbf{A} \cdot\left(\mathbf{D}_{H}(R)+s \mathbf{D}_{H}(L)\right) \\
& =\left[V_{1 / 2}^{e} \cdots V_{i+1 / 2}^{e} \cdots V_{N-1 / 2}^{e}\right]^{T} \\
& =\left[V^{e}\left(x_{1 / 2}\right) \cdots V^{e}\left(x_{i+1 / 2}\right) \cdots V^{e}\left(x_{N-1 / 2}\right)\right]^{T} \\
\mathbf{I}^{e} & =\mathbf{B} \cdot\left(\mathbf{D}_{I}(G)+s \mathbf{D}_{I}(C)\right) \\
& =\left[I_{0}^{e} \cdots I_{i}^{e} \cdots I_{N}^{e}\right]^{T} \\
& =\left[I^{e}\left(x_{0}\right) \cdots I^{e}\left(x_{i}\right) \cdots I^{e}\left(x_{N}\right)\right]^{T} \\
\mathbf{V}_{f}^{e} & =\mathbf{A} \cdot \mathbf{V}_{f} \\
& =\left[V_{f(1 / 2)}^{e} \cdots V_{f(i+1 / 2)}^{e} \cdots V_{f(N-1 / 2)}^{e}\right]^{T}, \\
& =\left[V_{f}^{e}\left(x_{1 / 2}\right) \cdots V_{f}^{e}\left(x_{i+1 / 2}\right) \cdots V_{f}^{e}\left(x_{N-1 / 2}\right)\right]^{T} \\
\mathbf{I}_{f}^{e} & =\mathbf{B} \cdot \mathbf{I}_{f} \\
& =\left[I_{f 0}^{e} \cdots I_{f i}^{e} \cdots I_{f N}^{e}\right]^{T} \\
& =\left[I_{f}^{e}\left(x_{0}\right) \cdots I_{f}^{e}\left(x_{i}\right) \cdots I_{f}^{e}\left(x_{N}\right)\right]^{T}
\end{aligned}
$$

then we obtain

$$
\begin{aligned}
V_{i+1}-V_{i} & =V_{i+1 / 2}^{e}+V_{f(i+1 / 2)}^{e} \\
I_{i+1 / 2}-I_{i-1 / 2} & =I_{i}^{e}+I_{f i}^{e} .
\end{aligned}
$$

Equation (73) is represented by the equivalent circuit shown as in Fig. 7.

Based on (71), the equivalent circuit model of MTLs can be similarly derived.

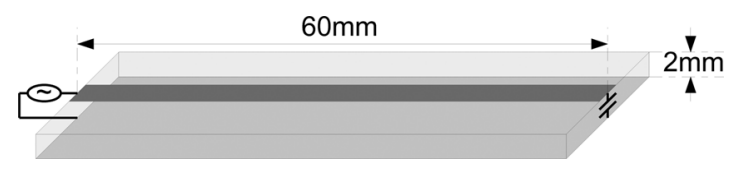

Fig. 8. Example for FDQ modeling accuracy.

\section{NUMERICAL EXPERIMENTS}

According to [19], the maximum frequency of interest is evaluated as

$$
f_{\max }=\frac{0.35}{t_{r}}
$$

where $t_{r}$ is the rise time of the input waveform. The maximum frequency determines the minimum wavelength within the spectral range of interest.

It is well established that for the finite difference method, a resolution of more than a dozen cells per wavelength is needed for required accuracy [11], which is also shown in Table I. In contrast, the FDQ schemes need much fewer cells per wavelength to achieve the same accuracy (see Table I), which approaches the Nyquist limit of two sample cells per wavelength. The intrinsic reason for the improvement is the global approximation. It is demonstrated in Table I that the more global the FDQ scheme is, the closer it approaches the Nyquist limit to achieve the required accuracy.

In large scale computation by using finite differencing, the key to improving computational efficiency is to reduce the grid points per wavelength while maintaining required accuracy. The FDQ method carries out the task of resolution reduction without loss of accuracy. On the other hand, the approximation globality is achieved at the cost of denser matrices. For example, both FDQ and FD modelings can result in the modified nodal analysis (MNA) equations as (55), but the difference is that $\mathbf{A}$ and $\mathbf{B}$ are tridiagonal, pentadiagonal, and so on matrices for (global) FDQ modeling, while they are simply diagonal matrices for (local) FD modeling. As very high order FDQ schemes not only are unnecessary, but also increase computational expense, the FDQ order usually should be no more than ten in practical application.

The first example discussed as follows is to test the FDQ modeling heuristic about the required resolutions as shown in Table I. In the circuit shown in Fig. 8, the length of the TL is $6 \mathrm{~cm}$, and the PUL parameters are $l=360 \mathrm{nH} / \mathrm{m}, c=100 \mathrm{pF} / \mathrm{m}$, $r=1 \mathrm{k} \Omega / \mathrm{m}$, and $g=1 \mathrm{mS} / \mathrm{m}$. The applied voltage source has an internal resistance of $100 \Omega$, and the load is a capacitance of $1 \mathrm{pF}$. We compare the frequency domain responses at the load capacitance by respectively applying the FD2, FDQ4, and FDQ6 schemes to the TL.

Assuming that the input trapezoidal signal has a rise/fall time of $25 \mathrm{ps}$, the maximum frequency is determined as $f_{\max }=$ $14 \mathrm{GHz}$ by using (74). The propagation velocity along the line is $v=1 / \sqrt{l c}=5 / 3 \times 10^{8} \mathrm{~m} / \mathrm{s}$; therefore, the minimum wavelength of interest is $\lambda_{\min }=v / f_{\max }=1.2 \mathrm{~cm}$ and the length of the TL is $6 \lambda_{\min }$.

First, we use the FD2, FDQ4, and FDQ6 schemes that have $1 \%$ phase error as in Table I. The numbers of cells required for 

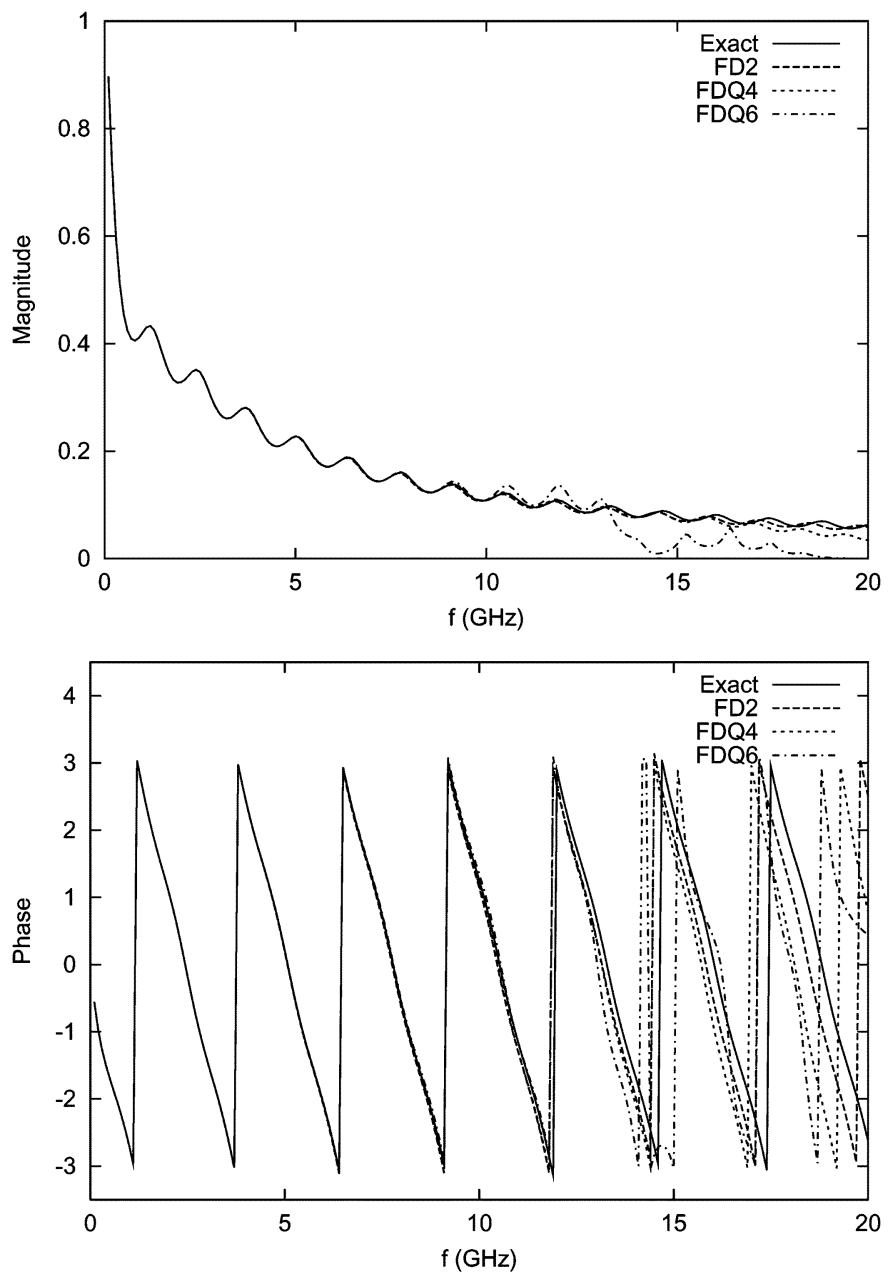

Fig. 9. Comparison of FDQ modeling accuracy at $1 \%$ phase error.

FD2, FDQ4, and FDQ6 are, respectively, 64, 23, and 17. The frequency domain responses are shown in Fig. 9. As expected, both the FD2 method and the FDQ method give agreeable results with the accurate value from dc to $14 \mathrm{GHz}$; however, the numbers of CPW for the FDQ methods are just a small fraction of that of the FD method.

If we use the FD2, FDQ4, and FDQ6 schemes that have 0.1\% phase error as in Table I, The numbers of cells required for FD2, FDQ4, and FDQ6 are, respectively, 204, 41, and 27. The frequency domain responses are shown in Fig. 10. All the results are agreeable from dc to more than $14 \mathrm{GHz}$, while the numbers of CPW for FDQ4 and FDQ6 are significantly reduced compared to that of the FD2 scheme.

The second example is about a microstrip TL on PCB carrying a supply voltage of $1.6 \mathrm{~V}$ to a VLSI chip. The equivalent circuit is shown as in Fig. 11(a). The PUL parameters of the TL are $l=340 \mathrm{nH} / \mathrm{m}, c=130 \mathrm{pF} / \mathrm{m}$, and $r=110 \Omega / \mathrm{m}$. The TL is illustrated by EM wave, shown as in Fig. 11(b), from a lightning $1 \mathrm{~km}$ away [20]. Note that the EM wave is composed of Gaussian pulses and high-frequency components. The pins and bonding wires are represented by inductance and capacitance [21], while the on-chip supply rails are represented by effective resistance and effective capacitance [22].
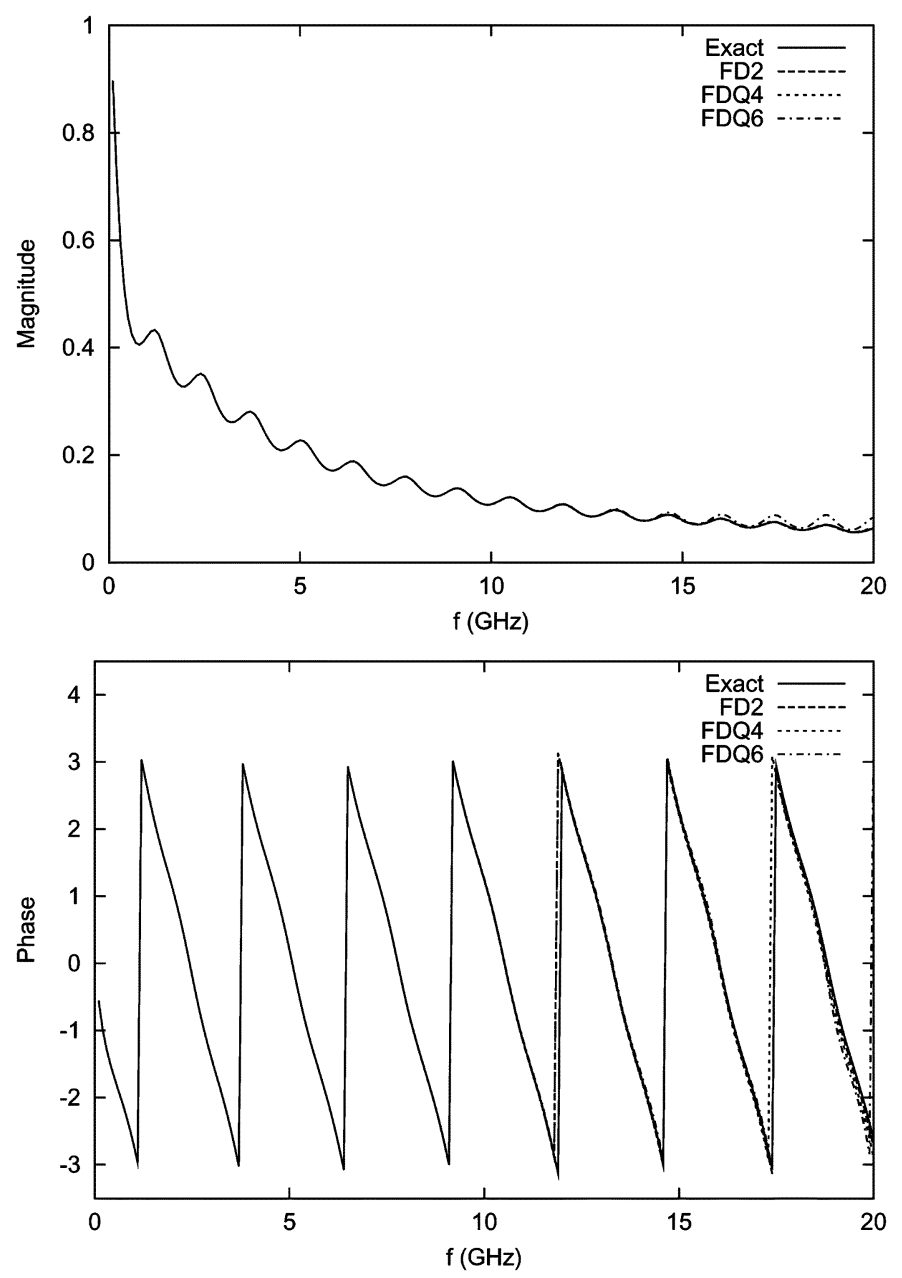

Fig. 10. Comparison of FDQ modeling accuracy at $0.1 \%$ phase error.

For the induced wave on the TL, the velocity is determined by the distributed inductance and capacitance as $v=1 / \sqrt{l c}=$ $1.5 \times 10^{8} \mathrm{~m} / \mathrm{s}$. Assuming that the maximum frequency of interest is $f_{\max }=50 \mathrm{GHz}$, the minimum wavelength is $\lambda_{\min }=$ $v / f_{\max }=3 \mathrm{~mm}$.

If the length of the TL is $3 \mathrm{~cm}$, then the number of minimum wavelength is 10 . If we use the original FDQ scheme by sampling two grid points per wavelength, the TL is segmented into 20 sections with 19 internal grid points. Consequently, there are 10 voltage differences and 11 current differences according to (46)-(49). The matrices $\mathbf{A}(10 \times 10)$ and $\mathbf{B}$ $(11 \times 11)$ are obtained by solving sets of $10 \times 10$ and $11 \times 11$ Vandermande equations, respectively. In this computation, the $10 \times 10$ and $11 \times 11$ Vandermande equations have condition numbers of $1.1558 \times 10^{8}$ and $8.8348 \times 10^{8}$, respectively.

If the length of the TL is $6 \mathrm{~cm}$, then the number of minimum wavelength is 20 . The TL needs to be segmented into $40 \mathrm{sec}-$ tions and the matrices $\mathbf{A}$ and $\mathbf{B}$ would have dimensions $20 \times 20$ and $21 \times 21$ if the straightforward process were followed. However, the $20 \times 20$ and $21 \times 21$ Vandermande equations have the condition numbers of $8.5457 \times 10^{16}$ and $4.2989 \times 10^{17}$, which are very ill-conditioned and, therefore, may lead to inaccurate 

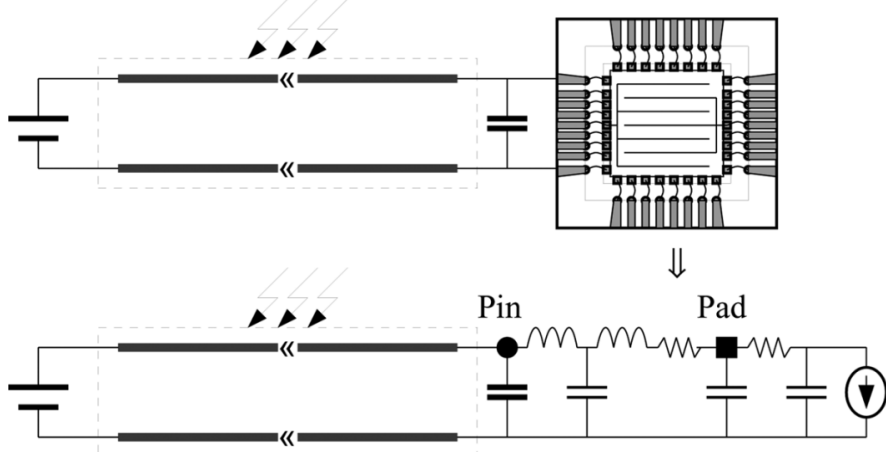

(a)

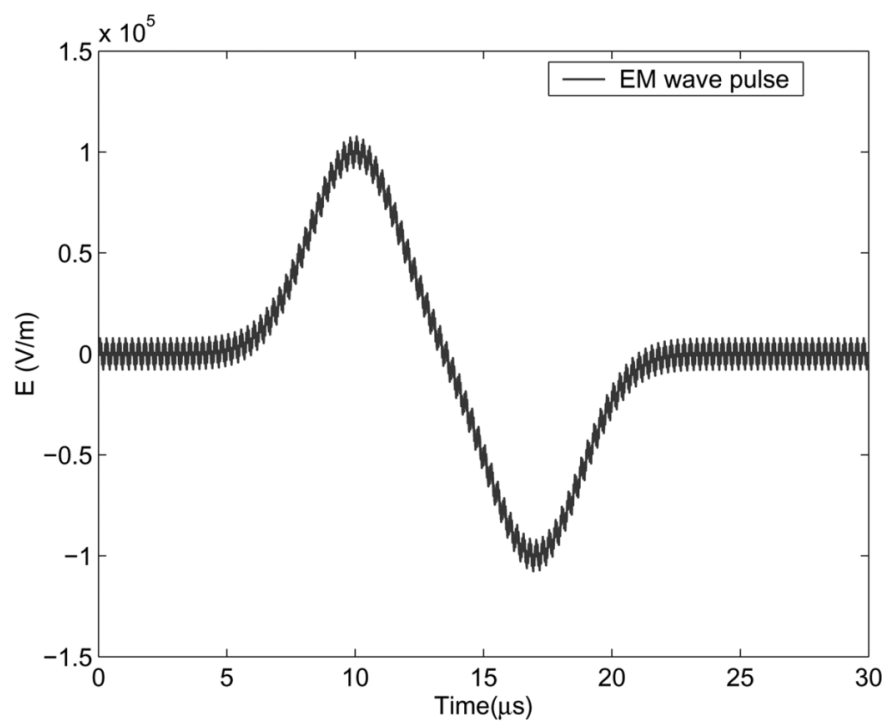

(b)

Fig. 11. Circuit of power supply TL with (a) EM wave illumination of (b) incident EM wave.

results. Nevertheless, the large dense matrices make the numerical computation inefficient as the computation effort increases on a cubic basis to handle dense matrices. In order to overcome the difficulties, the compact FDQ scheme FDQ6 are employed.

Let the EM wave have the propagation configuration as $\theta=$ $45^{\circ}, \theta_{E}=45^{\circ}$, and $\phi=-90^{\circ}$. We use the finite-differencequadrature time-domain (FDQTD) method to compute the previous problems. For simplicity, the backward Euler method is used for time-domain discretization. Fig. 12 shows the computing results for the TLs length of (a) 3, (b) 6 , and (c) $12 \mathrm{~cm}$, respectively. On an ULTRA-10 SUN workstation, it, respectively, takes $184.3,300.6$, and $675.6 \mathrm{~s}$ to perform the computations. It is demonstrated in Fig. 12 that the voltage supply at pins and pads can be affected significantly by the strong incident EM wave.

The next example is a TL carrying data from a function unit on PCB to the chip. The length of the TL is $2 \mathrm{~cm}$ and the TL has the same PUL parameters as in the first example. The circuit and the illuminating EM wave are shown in Fig. 13, respectively. Instead of performing the FDQ method in the time domain, we represent the TL using the equivalent circuit as shown in Fig. 7 and use HSPICE to simulate the entire circuit.

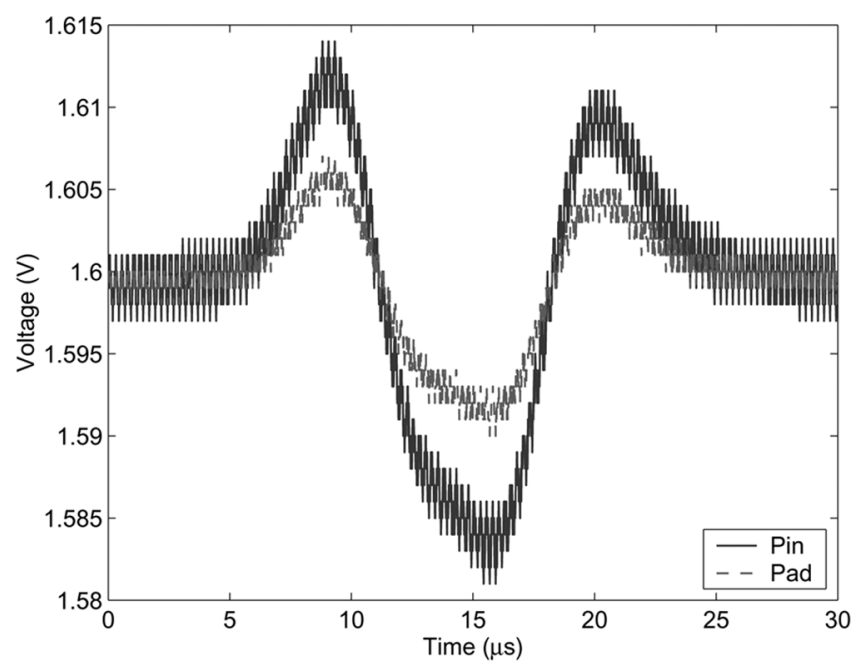

(a)

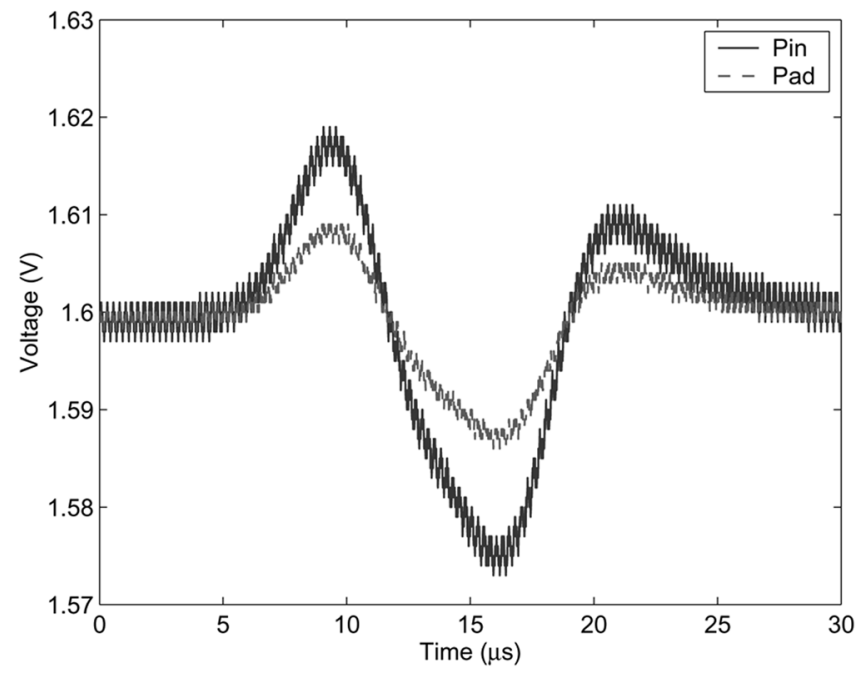

(b)

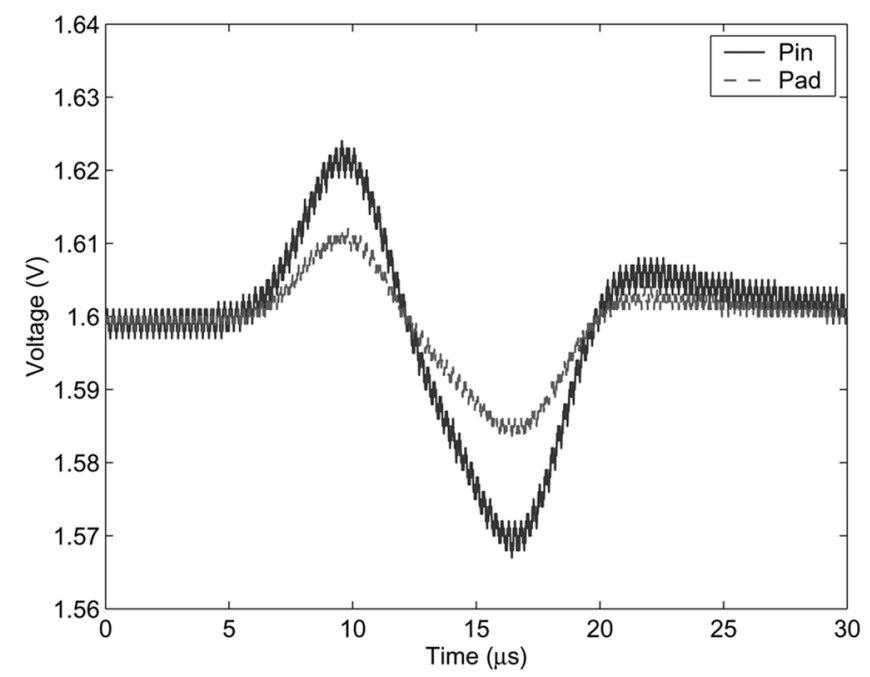

(c)

Fig. 12. Transient responses at the Pin and the Pad of the circuit in Fig. 11 with TL lengths (a) 3, (b) 6, and (c) $12 \mathrm{~cm}$, respectively.

The transmitted data have rise/fall times of $0.1 \mathrm{~ns}$. Assuming that the maximum frequency of interest is $20 \mathrm{GHz}$, the minimum 


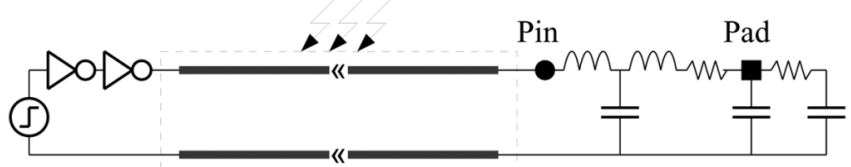

(a)

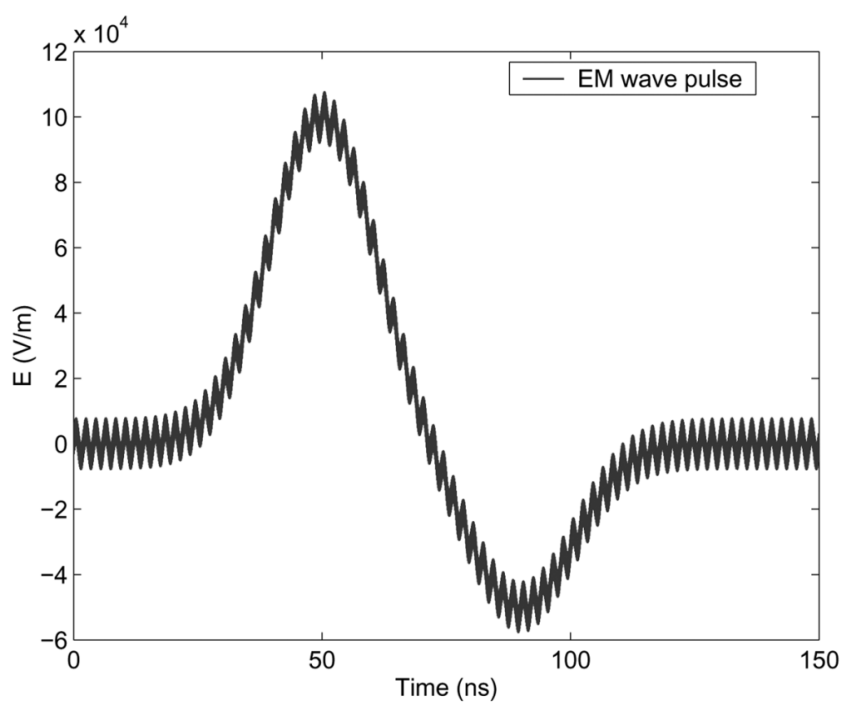

(b)

Fig. 13. Circuit of data transmission into chip through (a) TL with (b) incident EM wave.

wavelength is, therefore, $0.75 \mathrm{~cm}$. The FDQ6 scheme needs a resolution of 3.5 points per wavelength if the phase accuracy of $99 \%$ is required. The simulation results are shown in Fig. 14. In (a) are the waveforms at the pin and in (b) are those at the pad. The results show that the illuminating EM wave, especially the high-frequency components, can completely fail the data transmission through the TL.

\section{CONCLUSION}

An efficient numerical approximation technique, FDQ method, to proposed to model external field coupling to uniform or nonuniform TLs. A discrete modeling approach, FDQ adapts grid points along the transmission lines to compute the finite difference between adjacent grid points. Similarly to the Gaussian quadrature method to compute the numerical integrals, the FDQ method uses the global quadrature method to construct the approximation frames for the computation of numerical finite differences. To further reduce the computational expense of the FDQ method, the compact FDQ schemes are derived and their numerical dispersion is studied by using Fourier analysis. FDQ needs much sparser grid points than the finite difference (FD) methods do to achieve required accuracy. Equivalent voltage and current sources are derived, exciting the TLs at the grid points. Equivalent circuit models are therefore derived to represent the TLs illuminated by external electromagnetic waves. The FDQ-based equivalent models can be integrated into a simulator like SPICE. Numerical experiments

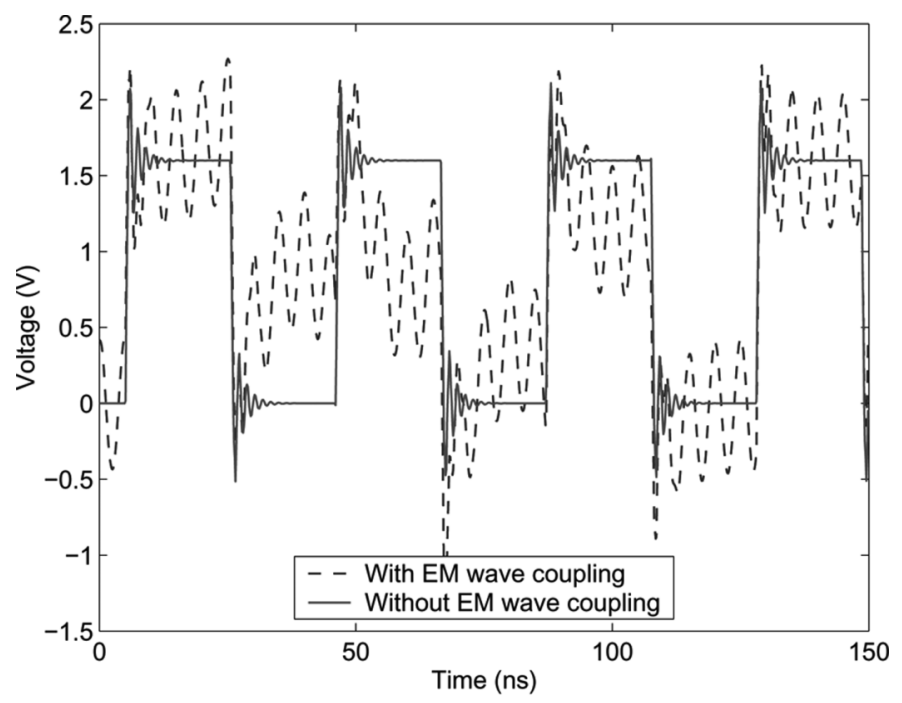

(a)

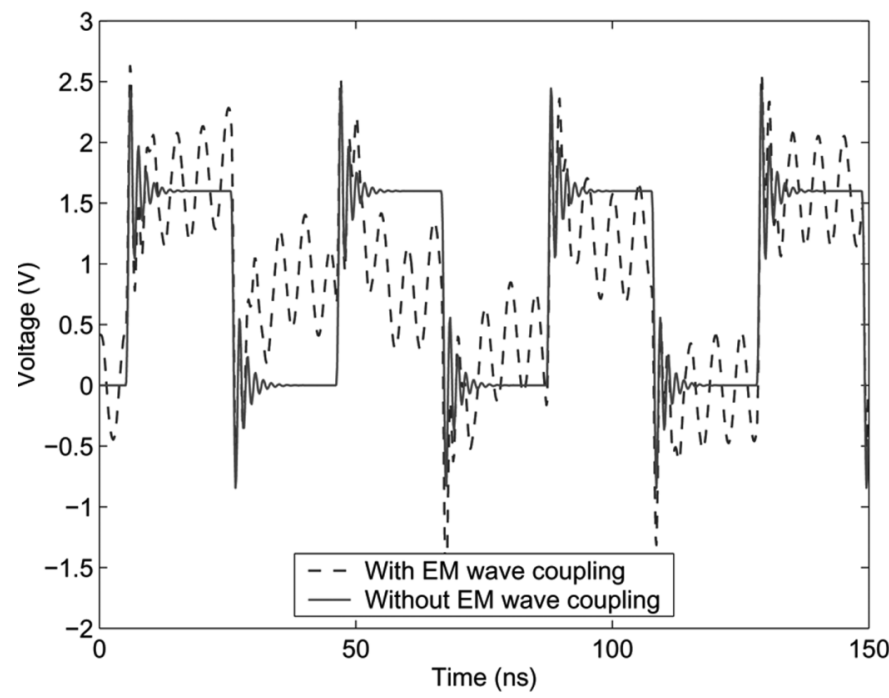

(b)

Fig. 14. Transient responses (a) at Pin and (b) at Pad of circuit in Fig. 13 with EM wave interference on TL.

show that FDQ-based modeling is an effective way to model the external EM wave coupling to the integrated circuits.

\section{REFERENCES}

[1] C. R. Paul, Analysis of Multiconductor Transmission Lines. New York: Wiley, 1994.

[2] K. S. Kuntz and R. J. Luebbers, The Finite Difference Time Domain Method for Electromagnetics. Boca Raton, FL: CRC Press, 1993.

[3] I. Wuyts and D. D. Zutter, "Circuit model for plan-wave incidence on multiconductor transmission lines," IEEE Trans. Electromagn. Compat., vol. 36, no. 3, pp. 206-212, 1994.

[4] I. Erdin, M. S. Nakhla, and R. Achar, "Circuit analysis of electromagnetic radiation and field coupling effects for networks with embedded full-wave modules," IEEE Trans. Electromagn. Compat., vol. 42, no. 4, pp. 449-460, Nov. 2000.

[5] M. Omid, Y. Kami, and M. Hayakawa, "Field coupling to nonuniform and uniform transmission lines," IEEE Trans. Electromagn. Compat., vol. 39, no. 3, pp. 201-211, Aug. 1997.

[6] T. Lapohos, J. LoVetri, and J. Seregelyi, "External field coupling to MTL networks with nonlinear junctions: Numerical modeling and experimental validation," IEEE Trans. Electromagn. Compat., vol. 42, no. 1, pp. 16-28, Feb. 2000. 
[7] C. R. Paul, "A SPICE model for multiconductor transmission lines excited by an incident electromagnetic field," IEEE Trans. Electromagn. Compat., vol. 36, no. 4, pp. 342-354, Nov. 1994.

[8] I. Erdin, A. Dounavis, R. Achar, and M. S. Nakhla, "A SPICE model for incident field coupling to lossy multiconductor transmission lines," IEEE Trans. Electromagn. Compat., vol. 43, no. 4, pp. 485-493, Nov. 2001.

[9] B. Fornberg, A Practical Guide to Pseudospectral Methods. London, U.K.: Cambridge Univ. Press, 1996.

[10] C. Canuto, Spectral Methods in Fluid Dynamics. New York: Springer-Verlag, 1988.

[11] M. N. O. Sadiku, Numerical Techniques in Electromagnetics. Boca Raton, FL: CRC Press, 2001.

[12] C. D. Taylor, R. S. Satterwhite, and C. W. Harrison, "The response of a terminated two-wire transmission line excited by a nonuniform electromagnetic field," IEEE Trans. Ant. Propag., vol. AP-13, no. 6, pp. 987-989, Nov. 1965.

[13] C. R. Paul, "Frequency response of multiconductor transmission lines illuminated by an electromagnetic field," IEEE Trans. Electromagn. Compat., vol. EMC-18, no. 4, pp. 183-190, Nov. 1976.

[14] A. K. Agrawal, H. J. Price, and S. H. Gurbaxami, "Transient response of multiconductor transmission lines excited by a nonuniform electromagnetic field," IEEE Trans. Electromagn. Compat., vol. EMC-22, no. 2, pp. 119-129, May 1980.

[15] Y. Kami and R. Sato, "Circuit-concept approach to externally excited transmission lines," IEEE Trans. Electromagn. Compat., vol. 27, no. 4, pp. 177-183, Nov. 1985.

[16] M. N. O. Sadiku, Elements of Electromagnetics. Fort Worth: Saunders College, 1989.

[17] E. Turkel, Advances in Computational Electrodynamics: The Finite-Difference Time-Domain Method, A. Taflove, Ed. Norwood, MA: Artech House, 1998.

[18] G. H. Golub and C. F. V. Loan, Matrix Computations. Baltimore, MD: The Johns Hopkins Univ. Press, 1996.

[19] Avant! Corporation, Fremont, CA 94538, "Star-HSPICE manual," 2000.

[20] D. R. MacGorman and W. D. Rust, The Electrical Nature of Storms. New York, NY: Oxford Univ. Press, 1998.

[21] J. M. Rabaey, Digital Integrated Circuits: A Design Perspective. Upper Saddle River, NJ: Prentice-Hall, 1996.

[22] R. Panda, D. Blaauw, R. Chaudhry, V. Zolotov, B. Young, and R. Ramaraju, "Model and analysis for combined package and on-chip power grid simulation," in Proc. ISLPED, 2000, pp. 179-184.

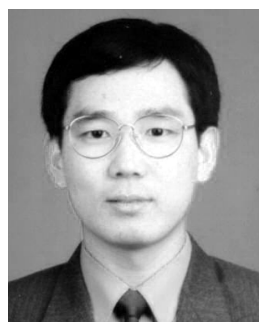

Qinwei Xu (S'02-M'07) was born in Shandong, China. He received the B.S. degree in electrical engineering from Shandong University, Shandong, China, the M.S. degree in electrical engineering from Shanghai Jiao Tong University, Shanghai, China, and the Ph.D. degree in computer science and engineering from the University of Michigan, Ann Arbor.

In 2004, he joined the Research and Development Team of Electronic Design Automation Tools, Cadence Design Systems, Inc., San Jose, CA. His research interests include interconnect modeling and transient simulation of VLSI chips, VLSI physical design and optimization, and numerical techniques in computational electromagnetics. He has authored over 30 papers on these topics in archival journals and international conference proceedings.

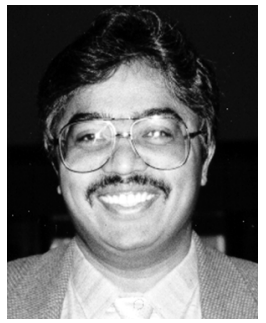

Pinaki Mazumder (S'84-M'88-SM'94-F'99) received the B.S.E.E. degree from the Indian Institute of Science, Bangalore, India, in 1976, the M.Sc. degree in computer science from the University of Alberta, Edmonton, AB, Canada, in 1985, and the $\mathrm{Ph} . \mathrm{D}$. degree in electrical and computer engineering from the University of Illinois, Urbana-Champaign, in 1987.

He is currently with the Department of Electrical Engineering and Computer Science, The University of Michigan, Ann Arbor. Prior to this, he spent two years as a Research Assistant with the Coordinated Science Laboratory, University of Illinois at Urbana-Champaign and over six years with Bharat Electronics Ltd. (a collaborator of RCA), Bangalore, India, where he developed several types of analog and digital integrated circuits for consumer electronics products. During the summer of 1985 and 1986, he was a Member of the Technical Staff of the Indian Hill Branch, AT\&T Bell Laboratories, Napperville, IL. From 1996 to 1997, he spent his sabbatical leave as a visiting faculty member with Stanford University, Stanford, CA, University of California, Berkeley, and Nippon Telephone and Telegraph, Atsugi-shi, Japan. His research interests include VLSI testing, physical design automation, and ultrafast circuit design. $\mathrm{He}$ has authored or coauthored over 160 papers on these topics in archival journals and proceedings of international conferences. He coauthored Testing and Testable Design of High-Density Random-Access Memories (Kluwer, 1996), Semiconductor Memories: Testing and Reliability (EEE Comput. Soc., 1998), and Genetic Algorithms for VLSI Design, Layout, and Test Automation (Prentice-Hall, 1998). He has two international patents pending. He has lead his research group's efforts in VLSI testing and built-in self-repair techniques and has developed silicon compilers for RAM, ROM, and programmable logic array (PLA) with built-in self-repairable capabilities. He has also done extensive work in the area of VLSI physical design. He developed a suite of distributed place-and-route tools for VLSI and field-programmable gate-array (FPGA) chips. For six years, he has been an Integrated Circuit Designer for semiconductor companies. He currently leads ultrafast circuit design activities for nano and quantum electronic devices. He has successfully developed computer-aided design (CAD) tools for high-performance VLSI circuit simulation (NDR SPICE) and numerous circuit topologies for quantum MOS and other quantum-well devices. Several U.S. and Japanese semiconductor companies, including Texas Instruments Incorporated, Hughes Research Laboratory, Lockheed-Martin, NTT, and NEC, have collaborated with him on this research. He was guest editor of the March 1993 "Special Issue on Multimegabit Memory Testing" of the IEEE Design and Test of Computers. He was the guest editor of two special issues on "Emerging Nanoelectronic Technologies and Their Applications" of the IEEE TRANSACTIONS ON VERY LARGE SCALE INTEGRATION (VLSI) SYSTEMS (December 1997) and the PROCEEDINGS OF THE IEEE (April 1998). He is on the Editorial Board of the PROCEEDINGS OF THE IEEE and is an Associate Editor of the IEEE TRANSACTIONS ON VERY LARGE SCALE INTEGRATION (VLSI) SYSTEMS.

Dr. Mazumder was a recipient of Digital's Incentives for Excellence Award, the BF Goodrich National Collegiate Invention Award, the National Science Foundation Research Initiation Award, and Bell Northern Research Laboratory Faculty Award. He was guest editor of the June 1994 "Special Issue On Memory Testing And Reliability" of the Journal of Electronic Testing Theory and Applications. He is a member of Sigma Xi, Phi Kappa Phi, and the Association for Computing Machinery (ACM) Special Interest Group on Design Automation (SIGDA). 